\title{
PRODUCTION ET ABONDANCE DE LA CIVELLE EN FRANCE AU DÉBUT DES ANNÉES 90.
}

\author{
G. CASTELNAUD*, D. GUERAULT ${ }^{\star \star}$, Y. DESAUNAY *^, P. ELIE
}

\begin{abstract}
* CEMAGREF de Bordeaux, Division Aquaculture et Pêche, 50 avenue de Verdun, B.P. 3, 33611 GAZINET Cedex, France.

** IFREMER Centre de Nantes, Direction «Ressources vivantes", rue de l'lle d'Yeu, B.P. 1049, 44037 NANTES Cedex 01, France.
\end{abstract}

\section{RÉSUMÉ}

La pêche de la civelle se pratique en France sur les façades de la Manche et de l'Atlantique, dans les cours d'eau affluant à la mer et sur certaines zones côtières adjacentes. Cette activité a commencé à s'intensifier à la fin des années 60 avec l'apparition de la technique du drossage et l'augmentation du nombre de pratiquants, professionnels et non-professionnels, liée à l'augmentation des prix d'achat de la civelle par les Espagnols.

Les enregistrements statistiques de capture et d'effort de pêche des administrations sont souvent fragmentaires et peu fiables.

Ce travail a donc consisté à réaliser, à partir des données officielles disponibles et des résultats des enquêtes et systèmes de suivi des pêcheries de civelles développés par I'IFREMER et le CEMAGREF depuis la fin des années 70 , un bilan de l'exploitation pour l'année de référence 1989 et un diagnostic sur les tendances de l'abondance des civelles.

4360 pêcheurs dont 1160 pêcheurs professionnels (27\%) et 3200 nonprofessionnels $(73 \%)$ ont débarqué 520 tonnes de civelles en 1989, réalisant un chiffre d'affaires de plus de 200 millions de francs. La part des non-professionnels était de $33 \%$ soit 170 tonnes et 68 millions de francs.

L'analyse des séries chronologiques d'indicateurs halieutiques obtenues sur les bassins de la Vilaine, de la Loire et de la Gironde, autorise à penser qu'on a assisté au début des années 80 , à une chute de l'abondance des flux de civelles, qui ne s'est pas démentie depuis.

La pertinence et les limites des résultats sont discutées et un certain nombre de mesures concernant la ressource et l'activité de pêche sont proposées.

Mots-clés : Anguille, Anguilla anguilla L., estuaire, pêche artisanale, techniques de pêche, réglementation, abondance, production.

\section{YIELD AND ABUNDANCE OF THE CIVELLE (ANGUILLA ANGUILLA L.) AT THE BEGINNING OF THE NINETIES.}

\begin{abstract}
The civelle fishery is carried out in the lower sections of the rivers that flow into the Bay of Biscay and the Channel together with a small part of the adjacent coast around the mouth of these rivers. This activity began to develop rapidly at the end of the sixties in connection with the use of push nets and the increase in the Spanish market prices for civelle.
\end{abstract}


The official statistical data on fish catches and fishing effort registered is often unreliable and fragmentary. Consequently, this study has tried to establish the amount of fishing over one recent year (1989) and a diagnosis of the abundance of civelle, using the official data available and the result of the investigations carried out by CEMAGREF and IFREMER.

A total of 4360 fishermen made up of 1160 professionals (27\%) and 3200 nonprofessionals (73\%) landed 520 tons of civelle in 1989 with a turn-over of 200 millions F.F. The proportion produced by the non-professional fishermen amounted 170 tons and 68 millions F.F. (33 \%).

The analysis of time series of fishing indicators obtained from the river basins of Vilaine, Loire and Gironde, led to the conclusion that the abundance of civelle has fallen dramatically at the beginning of the eighties. There has been no change in the situation since then.

The relevance and the accuracy of these results are discussed and some proposals are made concerning the biological resource and the fishing activity.

Key-words : Eel, Anguilla anguilla L., estuary, artisanal fishery, fishing techniques, regulations, abundance, production.

\section{INTRODUCTION}

La pêche de la civelle en France s'exerce sur les parties basses des cours d'eau exoréiques et sur certaines zones côtières adjacentes.

Sur les côtes, la pêche est sous réglementation maritime. Dans les cours d'eau affluant à la mer, la pêche est sous réglementation maritime dans les estuaires à l'aval de la limite de salure des eaux et sous réglementation fluviale à l'amont (Fig.1).

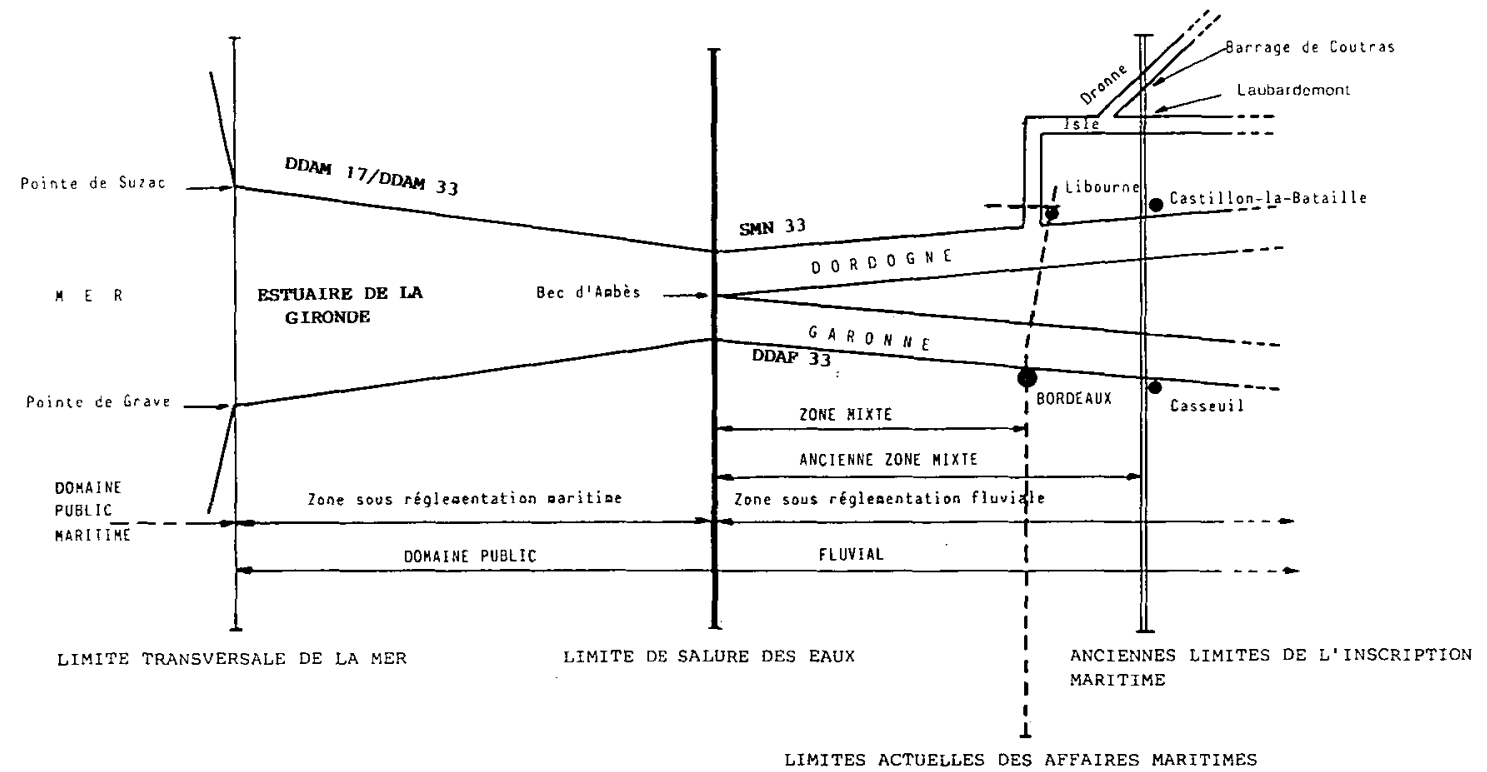

Figure 1 : Compartimentage administratif et réglementaire des cours d'eau affluant à la mer : exemple du bassin de la Gironde.

Figure 1 : Statutory of rivers connected with the ocean : example from the Gironde basin. 
Cinq catégories de pêcheurs cohabitent : les marins-pêcheurs professionnels à pied ou en bateau qui ont un droit exclusif de pêche dans les estuaires sous réglementation maritime et dont une partie a conservé des droits de pêche dans les zones mixtes à l'amont de la limite de salure des eaux; les pêcheurs professionnels en eau douce, qui se cantonnent dans les eaux sous réglementation fluviale ; les amateurs maritimes à pied ; les amateurs fluviaux à pied ou en bateau ; les braconniers avec ou sans droit de pêche qui commercialisent leurs prises.

II n'existe pas de centralisation de la vente des civelles dans des systèmes de type criée et les enregistrements des captures sont basés sur les déclarations des pêcheurs professionnels et des mareyeurs. Compte tenu de la situation de concurrence des différentes catégories de pêcheurs, de l'intérêt financier de la pêche de la civelle (qui peut s'effectuer avec un investissement limité dans de nombreux sites) et de la complexité du découpage administratif et réglementaire, les données d'effort et de production obtenues auprès des différents services gestionnaires DDAM (1), DDAF (2), SMN (3) ou du CIPE (4), sont souvent fragmentaires et donc non utilisables pour réaliser un suivi précis de l'évolution de la ressource.

De ce fait, sur quelques secteurs et parfois pour des durées limitées, certains organismes de recherche ont mis en place leurs propres systèmes d'inventaire et de suivi des pêcheries à des fins socio-économiques et biologiques.

La capture de civelles est attestée dès le XIXe siècle en France (CASTELNAUD, 1978 ; ELIE, 1979 ; GASCUEL, 1987). Sa pêche était interdite dans les eaux sous réglementation fluviale au début du XXe siècle (DAUBRÉE et DE DROUIN DE BOUVILLE, 1900 ) et c'est à cette époque qu'elle a commencé à se développer sur la façade atlantique.

L'installation de la pêcherie actuelle s'est opérée en quatre phases :

- De 1920 à 1950 environ, la pêche s'effectuait en zones maritime et fluviale uniquement le long des berges, à pied ou en bateau à l'arrêt, au tamis à main d'un diamètre généralement inférieur à $0,8 \mathrm{~m}$. Le pêcheur repérait les cordons de civelles et les attirait avec une source lumineuse (ELIE, 1979). Cette technique est toujours utilisée surtout en zone fluviale, où les cordons de migration se forment de façon privilégiée.

- Une seconde phase, qui a duré jusqu'en 1970 environ, s'est amorcée dans les années 50 avec l'apparition dans les estuaires sous réglementation maritime de la technique de drossage, qui consiste à pousser un tamis circulaire ou rectangulaire de chaque côté du bateau (ELIE, 1979). La largeur du tamis a été fixée ultérieurement à 1,20 $\mathrm{m}$ par l'arrêté $\left(n^{\circ} 758\right.$ MMP1) du 17 février 1965.

En zone sous réglementation fluviale, le diamètre du tamis avait été porté à $1,20 \mathrm{~m}$ par le décret du 16 septembre 1958 ; il restait manoeuvré à la main. En exécution des dispositions de ce décret, la pêche de la civelle avait été autorisée dans certains cours d'eau d'llle-et-Vilaine, du Morbihan, de la Loire-Atlantique, de la Vendée, de la CharenteMaritime, de la Gironde, de la Dordogne, des Landes et des Pyrénées-Atlantiques. Les dimensions du tamis avaient été fixées à 0,60 $\mathrm{m}$ dans l'llle-et-Vilaine, le Morbihan, la Vendée, la Charente-Maritime, à $0,80 \mathrm{~m}$ dans la Dordogne, à $1,00 \mathrm{~m}$ dans la Gironde et à $1,20 \mathrm{~m}$ dans la Loire-Atiantique, les Landes et les Pyrénées-Atlantiques (POPELIN, 1971).

L'évolution des techniques de pêche et des réglementations a entraîné une augmentation du nombre de pratiquants qui a été amplifiée à la fin des années 60 par les prix d'achat de la civelle offerts par les négociants espagnols (ceux-ci ont triplé en francs constants 1990 entre 1969 et 1971, passant en francs courants de 6,25 F à $19 \mathrm{~F}$ par $\mathrm{kg}$ en moyenne) en liaison avec une demande importante de civelle vivante sur le marché international (ELIE et FONTENELLE, 1982).

(1) DDAM : Direction Départemantale des Affaires Maritimes ;

(2) DDAF : Direction Départementale de l'Agriculture et de la Forêt ;

(3) SMN : Service Maritime et de Navigation ;

(4) CIPE : Comité Interprofessionnel des Poissons Migrateurs des Estuaires, créé par l'arrêté du 24 avril 1978 modifié, devenu Comité Interprofessionnel des Poissons Migrateurs et des Estuaires (Arrêtés ministériels du 7 août 1987 et du 15 février 1991). 
En 1970, POPELIN (1971) évaluait à partir d'une enquête administrative la production totale à 1345 tonnes pour une valeur de 18 millions de francs (Tableau I) ; 648 marins-pêcheurs professionnels et 2424 pêcheurs fluviaux professionnels et amateurs ont été dénombrés.

Tableau I : Estimations de la production (en tonnes) et du nombre de pêcheurs entre 1970 et 1986.

(1) ajouter nombre inconnu de pêcheurs à pied et de braconniers maritimes évalué à plus de 3500 en 1976 (sauf DESAUNAY et AUBRUN, 1988).

(2) ajouter nombre inconnu de braconniers et de pêcheurs sur le Domaine Privé.

(3) comprenant pêcheurs maritimes à pied, professionnels et amateurs fluviaux.

Table I : Production estimates (in tons) and number of fishermen between 1970 and 1986.

(1) unknown number of marine pedestrian fishermen and poachers to be added : more than 3500 in 1976 (DESAUNAY and AUBRUN, 1988, non concerned).

(2) unknown number of poachers and fishermen on the Private Domain to be added.

(3) including marine pedestrian river fishermen, professional and amateur river fishermen.

\begin{tabular}{|c|c|c|c|c|c|c|}
\hline & \multicolumn{6}{|c|}{ ANNÉES } \\
\hline & 1970 & 1976 & 1979 & 1983 & & 1986 \\
\hline Production (marins-pêcheurs) & 450 & 1455 & 1175 & 591 & 260 & \\
\hline Production (pêcheurs fluviaux) & 895 & & 675 & & & \\
\hline Production totale & 1345 & & 1850 & & & 500 \\
\hline Prix moyen au Kg (Francs courants) & 14 & 24 & 37 & 130 & 164 & \\
\hline $\begin{array}{l}\text { Valeur totale } \\
\text { (Millions de Francs courants) }\end{array}$ & 18 & 32 & 68.5 & 77 & 43 & 82 \\
\hline Nombre de marins-pêcheurs (1) & 648 & 1850 & 964 & 995 & 677 & 850 \\
\hline Nombre de pêcheurs fluviaux (2) & 2424 & & 2588 & & & $4000(3)$ \\
\hline Origine des données & $\begin{array}{l}\text { POPELIN } \\
(1971)\end{array}$ & $\begin{array}{c}\text { ELIE } \\
(1979)\end{array}$ & $\begin{array}{l}\text { C.I.P.E. } \\
\text { (1982) }\end{array}$ & $\begin{array}{l}\text { C.I.P.E. } \\
(1983)\end{array}$ & $\begin{array}{l}\text { C.I.P.E. } \\
(1986)\end{array}$ & $\begin{array}{l}\text { DESAUNAY ET } \\
\text { AUBRUN (1988) }\end{array}$ \\
\hline
\end{tabular}

- L'augmentation de l'effort de pêche total et des captures totales, déjà bien engagée avant 1970, a déterminé une troisième phase qui a culminé en 1979 après l'apparition du pibalour dans les estuaires maritimes de Charente, Seudre et Gironde. Le pibalour constitue une adaptation locale de la technique du drossage avec les tamis, jugée trop peu performante dans ces estuaires. Les filets-poches sont supportés par deux cadres placés sur les côtés ou à l'avant du bateau.

L'arrêté du 23 décembre 1975 prévoit que la surface de chaque filet ne doit pas dépasser $7 \mathrm{~m}^{2}$; le tonnage du bateau doit être égal ou inférieur à 10 tonneaux et la puissance du moteur doit être égale ou inférieure à $60 \mathrm{CV}$.

A partir de 1979, pour pratiquer la pêche de la civelle, tout marin-pêcheur professionnel a dû obtenir un droit de pêche annuel délivré par l'organisation interprofessionnelle (CIPE). L'obtention de ce droit de pêche est subordonnée à certaines conditions de professionnalisme et d'exploitation qui, moins limitatives que pour le pibalour, 
s'appliquent à la pêche au tamis : navire de moins de 10 tonneaux de jauge brute, longueur de bateau inférieure à $12 \mathrm{~m}$ et puissance du moteur au maximum égale à $100 \mathrm{CV}$. Ces conditions n'ont guère varié depuis 1979 et sont reconduites chaque année.

La production totale de l'année 1979 a été estimée après enquête à 1850 tonnes pour une valeur de 68,5 millions de francs par le CIPE (1982), qui indiquait que 1979 a été choisie comme référence car il s'agissait de la dernière bonne année de pêche. 964 marins-pêcheurs et 2588 pêcheurs professionnels et amateurs fluviaux étaient dénombrés (Tableau I).

- Après cette montée en puissance dans la décade 1970-1979, une quatrième phase s'est annoncée au début des années 80 avec un fort déclin des apports totaux, illustré par les données concernant les marins-pêcheurs, les seules en notre possession. Alors que pour l'année 1976, ELIE (1979) était arrivé au chiffre minimum de 1455 tonnes pour une valeur de 32 millions de francs, débarquées par 1850 marins-pêcheurs, le CIPE (1983) évaluait après correction des chiffres collectés auprès des pêcheurs, la production pour l'année 1983 à 591 tonnes, sa valeur à 77 millions de francs et le nombre de marins-pêcheurs à 995 . Les chiffres non corrigés produits antérieurement par cet organisme pour les années 1980, 1981, 1982, se sont avérés proches de ces derniers en ce qui concerne le tonnage et le nombre de pêcheurs.

L'activité de pêche à la civelle a continué à mobiliser les pêcheurs à cause des chiffres d'affaires réalisables : les prix d'achat au pêcheur étaient restés stables en francs constants entre 1971 et 1979 ; ils avaient doublé entre 1979 et 1983 en francs constants, passant en 4 ans en francs courants de $37 \mathrm{~F}$ à $130 \mathrm{~F}$ en moyenne par kilogramme (Tableau I).

Les derniers chiffres globaux produits depuis 1983 concernaient la seule année 1986 et avaient deux origines (Tableau I) : le CIPE (1986) pour les seuls marins-pêcheurs et I'IFREMER (DESAUNAY et AUBRUN, 1988) pour l'ensemble des pêcheurs.

Le niveau des apports et de l'effort total de l'année 1986 semble s'être maintenu jusqu'à aujourd'hui, sans être guère affecté par les changements de réglementation intervenus depuis, notamment en zone fluviale où à partir de 1986 la dimension du tamis amateur a été réduite à $50 \mathrm{~cm}$, avec interdiction de vendre les produits de la pêche.

Face aux lacunes et à la disparité des données accessibles, nous allons tenter, dans un premier temps, de vérifier cette évolution, en dressant un bilan de l'exploitation pour l'année de référence 1989 en termes d'effort total (nominal) et de production et, dans un deuxième temps, de présenter les tendances de l'abondance qui se dégagent de l'analyse des séries chronologiques d'indicateurs halieutiques obtenues sur les bassins de la Vilaine, de la Loire et de la Gironde.

\section{MATÉRIEL ET MÉTHODE}

La méthode a consisté à utiliser le matériel disponible sur l'exploitation de la civelle provenant d'une part des enregistrements des services gestionnaires, des organisations de pêcheurs et d'autre part des enquêtes et des systèmes de suivi développés par l'IFREMER et le CEMAGREF depuis la fin des années 70.

\section{Les enquêtes}

Un inventaire de l'exploitation de l'anguille pour ses trois écophases : civelle, anguille jaune et anguille argentée, a débuté en 1985 par une enquête par correspondance auprès des services administratifs chargés de la pêche, des organisations professionnelles, et des laboratoires côtiers. Elle a été suivie par des enquêtes de terrain menées sur le littoral Bretagne en février et mars 1986 (AUBRUN, 1986), sur le littoral Sud-Gascogne de septembre à novembre 1986 (AUBRUN, 1987) et sur le littoral Manche-Est en février et mars 1987 (DESAUNAY, 1987). Pour le Lay et le bassin de la Sèvre Niortaise, (AUBRUN, 1987) a repris les travaux de GASCUEL (1987) basés sur le dépouillement de carnets de pêche et sur des vérifications de terrain. Cet inventaire était plutôt axé 'sur la pêche maritime. II a apporté des informations originales sur l'ensemble des sites de pêche, sur l'effort nominal (nombre de pêcheurs) et la production des quatre catégories officielles d'exploitants dans les secteurs autres que les grands estuaires, pour l'année 1986. 
Une étude nationale de la pêche professionnelle fluviale et lacustre (CASTELNAUD et BABIN, $1990 ; 1992$ ) a permis de recenser les zones de pêche à la civelle et les effectifs de pêcheurs professionnels en eau douce, pratiquant cette pêche en 1989. Cette étude a consisté en une revue bibliographique, une enquête administrative et des enquêtes de terrain organisées par bassins versants et par secteurs de pêche homogènes. Des entretiens pour partie directifs s'appuyant sur un questionnaire détaillé, ont été menés avec l'ensemble des pêcheurs professionnels ou un échantillon selon les secteurs. D'autres intervenants tels que poissonniers, mareyeurs, gardes-pêche, ont aussi été interviewés.

Les enquêtes de terrain ont été doublées en 1990 d'une enquête par courrier auprès des pêcheurs professionnels, axée plus particulièrement sur les productions. Un taux de réponses de $40 \%$ a été obtenu, et le redressement de l'échantillon effectué sur la base d'une typologie des pêcheurs professionnels, a permis d'estimer les productions de l'année 1989, par bassin et par espèce ou écophase d'intérêt halieutique, dont la civelle (BABIN, 1993).

\section{Les suivis de pêcheries}

\subsection{Bassins de la Vilaine et de la Loire}

Les statistiques officielles rendent compte de l'évolution des captures de 1977 à 1990 des marins-pêcheurs et des professionnels fluviaux en Loire et de celles des marinspêcheurs en Vilaine (ELIE et RIGAUD, 1984 ; GUERAULT et al., 1994). Elles ne sont jamais associées à des données concernant l'effort de pêche des professionnels et ne renseignent pas sur l'effort de pêche et les captures des non-professionnels.

Des indices d'efforts de pêche et de production d'une part, des prises par unité d'effort (P.U.E.) d'autre part, ont été progressivement acquis au niveau du réseau commercial pour :

- une sélection d'un nombre variable de marins-pêcheurs travaillant en zone maritime (13 à 69 en Loire, 38 à 65 en Vilaine) établie sur la base d'une assiduité minimale saisonnière de 25 jours de pêche de 1977 à 1990 dans les deux estuaires ;

- un lot témoin de marins-pêcheurs travaillant en zone maritime ( 9 en Loire, 4 en Vilaine) continuellement présents et de manière très assidue ( $>40$ sorties en Loire et $>70$ sorties en Vilaine) de 1977 à 1990 en Loire et de 1979 à 1990 en Vilaine (GUERAULT et DESAUNAY, 1989).

Ces données issues d'une sélection et d'un lot témoin de pêcheurs dont la représentativité n'est pas déterminée, ne peuvent donner lieu à des évaluations de l'effort total et de la production totale, mais elles informent sur leurs tendances.

Un suivi plus précis des pêcheries a permis d'effectuer de 1985 à 1990 :

- le dénombrement des effectifs de pêcheurs professionnels, marins et fluviaux par identification des navires actifs sur la pêcherie ;

- le recueil de données individuelles à l'échelle d'une sortie $(\mathrm{kg} / \mathrm{sortie})$ et de la totalité de la saison de pêche (poids et nombre de sorties par saison) d'un échantillon de pêcheurs professionnels maritimes et fluviaux dans le réseau commercial local (taux d'échantillonnage variant de 30 à $50 \%$ de la population totale de pêcheurs professionnels).

Ces deux séries de données sont à l'origine pour la période 1985 - 1990, de l'évaluation de la production (C) en $\mathrm{kg}$, de l'effort de pêche (f) en nombre de sorties et de la prise par unité d'effort (P.U.E.) en kg par sortie des marins-pêcheurs (uniques exploitants) en Vilaine et des marins-pêcheurs et professionnels fluviaux en Loire (GUERAULT et al., 1993). Ces indicateurs halieutiques sont exprimés avec un risque statistique de $5 \%$ (échantillonnage simple en Vilaine, échantillonnage stratifié par catégorie de pêcheurs professionnels en Loire).

\subsection{Bassin de la Gironde}

Les données de capture et d'effort journalières ou saisonnières proviennent pour la période 1978 à 1982 de carnets de pêche distribués à un groupe de pêcheurs professionnels marins et fluviaux (CASTELNAUD et al., 1981) et de cahiers de pêches 
personnels de certains pêcheurs (CANTRELLE, 1981). Le nombre total de carnets et de cahiers de pêche a varié de 23 à 38 selon les années, pendant cette période (taux d'échantillonnage variant de 7 à $12 \%$ ). Pour la période $1983-1992$, nous avons créé un réseau de pêcheurs coopératifs selon la technique de l'échantillonnage stratifié par métier et par zone de pêche. Ces pêcheurs coopératifs sont fidélisés d'une année sur l'autre ; ils nous fournissent des données précises de captures en poids par sortie ou par jour de pêche et des renseignements complémentaires, généralement issus directement de leur cahier de pêche personnel. Leur nombre a varié de 13 à 27 selon les années pendant cette période (taux d'échantillonnage progressant de 6 à $20 \%$ ).

Deux métiers ont été différenciés : le métier civelle-pibalour, exercé uniquement par des marins-pêcheurs dans l'estuaire maritime de Gironde durant toute la période 1979 - 1992 ; le métier civelle-tamis exercé par des pêcheurs professionnels marins et fluviaux, concurremment avec des pêcheurs non-professionnels dans les zones mixtes de Garonne - Dordogne - Isle durant toute la période 1979 - 1992. Le métier civelle-tamis a aussi été pratiqué par un petit nombre de marins-pêcheurs dans l'estuaire maritime jusqu'en 1988 inclus.

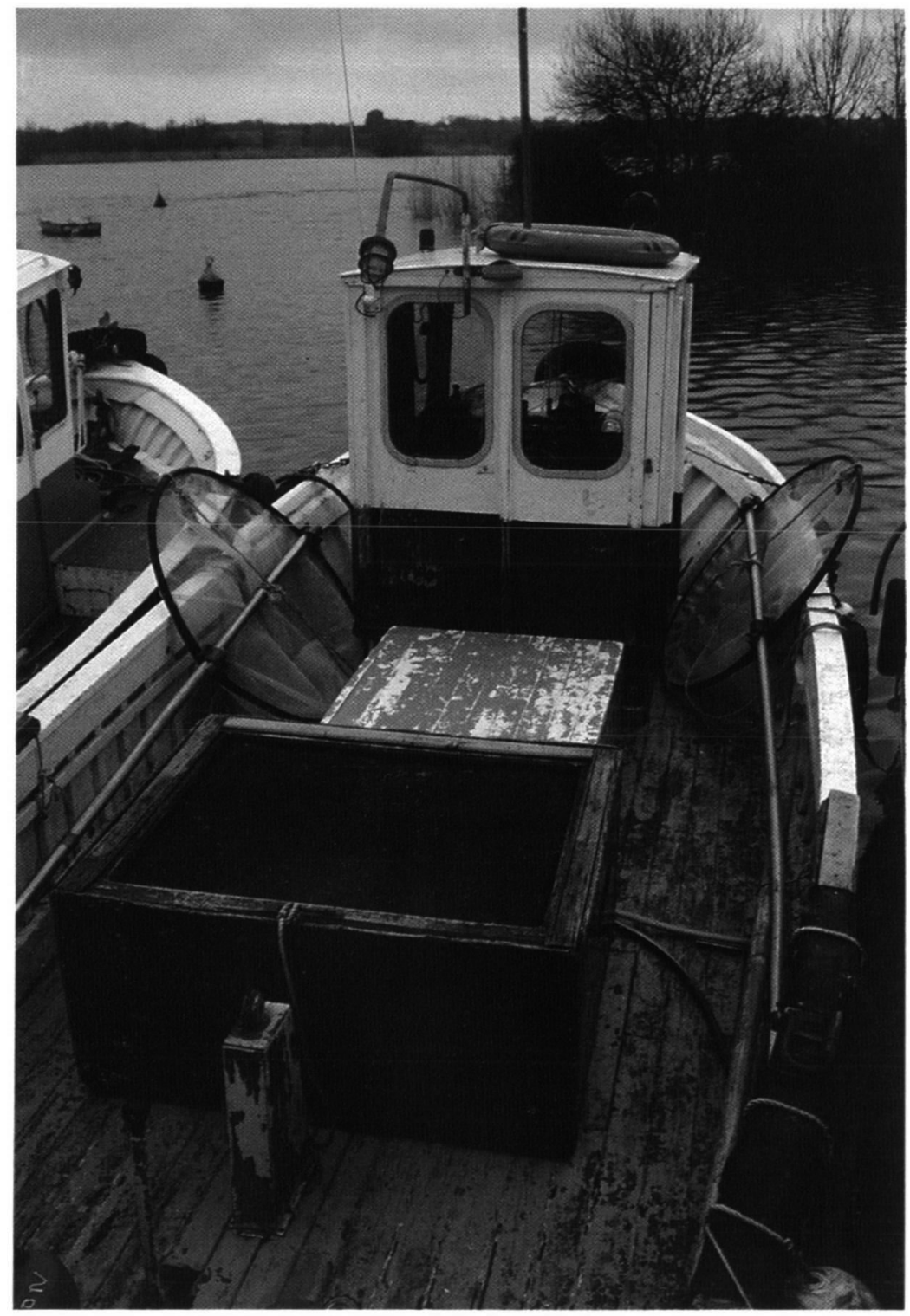

Tamis circulaire pour la pêche de la civelle dans l'estuaire de la Loire. 


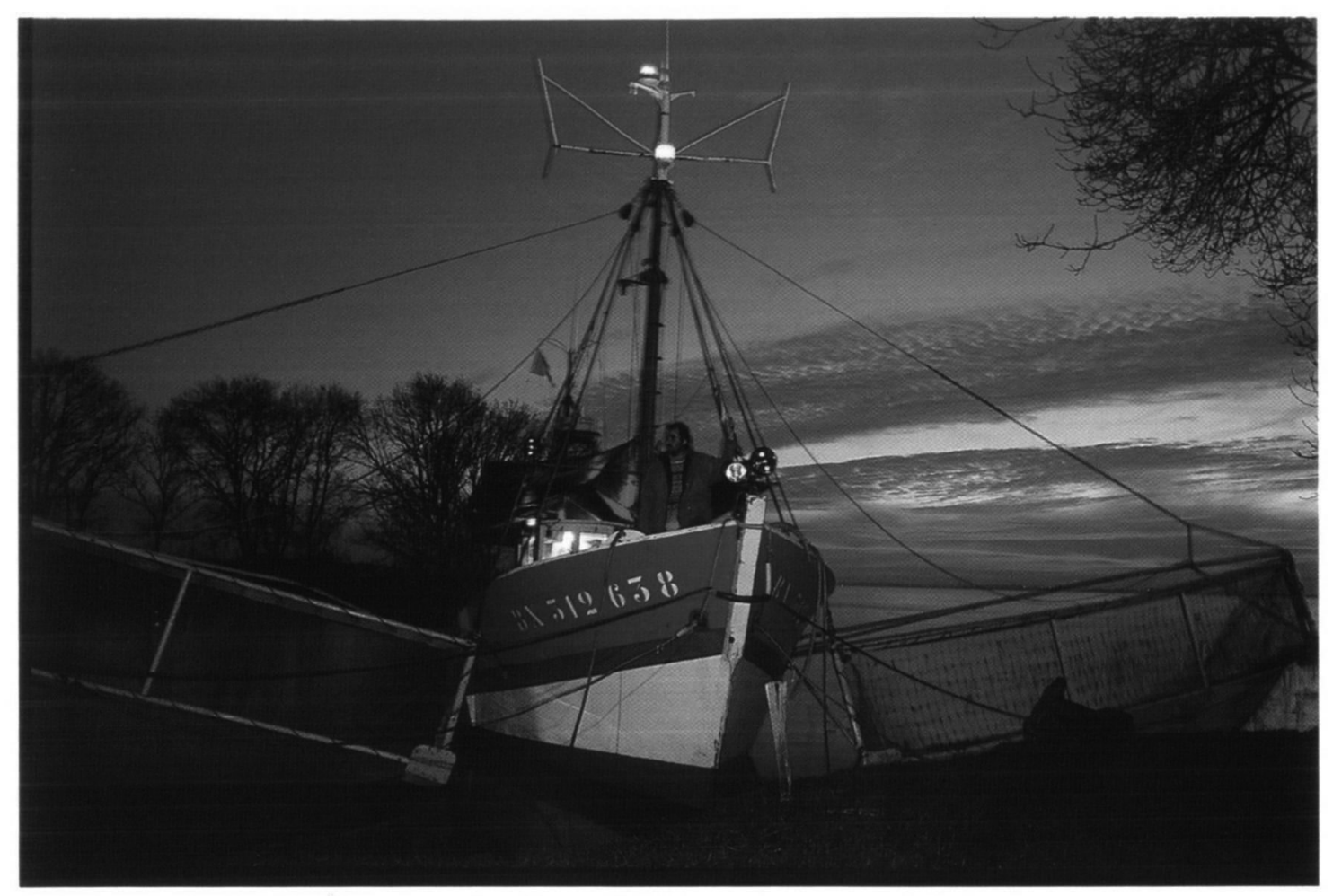

Pilabour pour la pêche de la civelle dans l'estuaire de la Gironde.

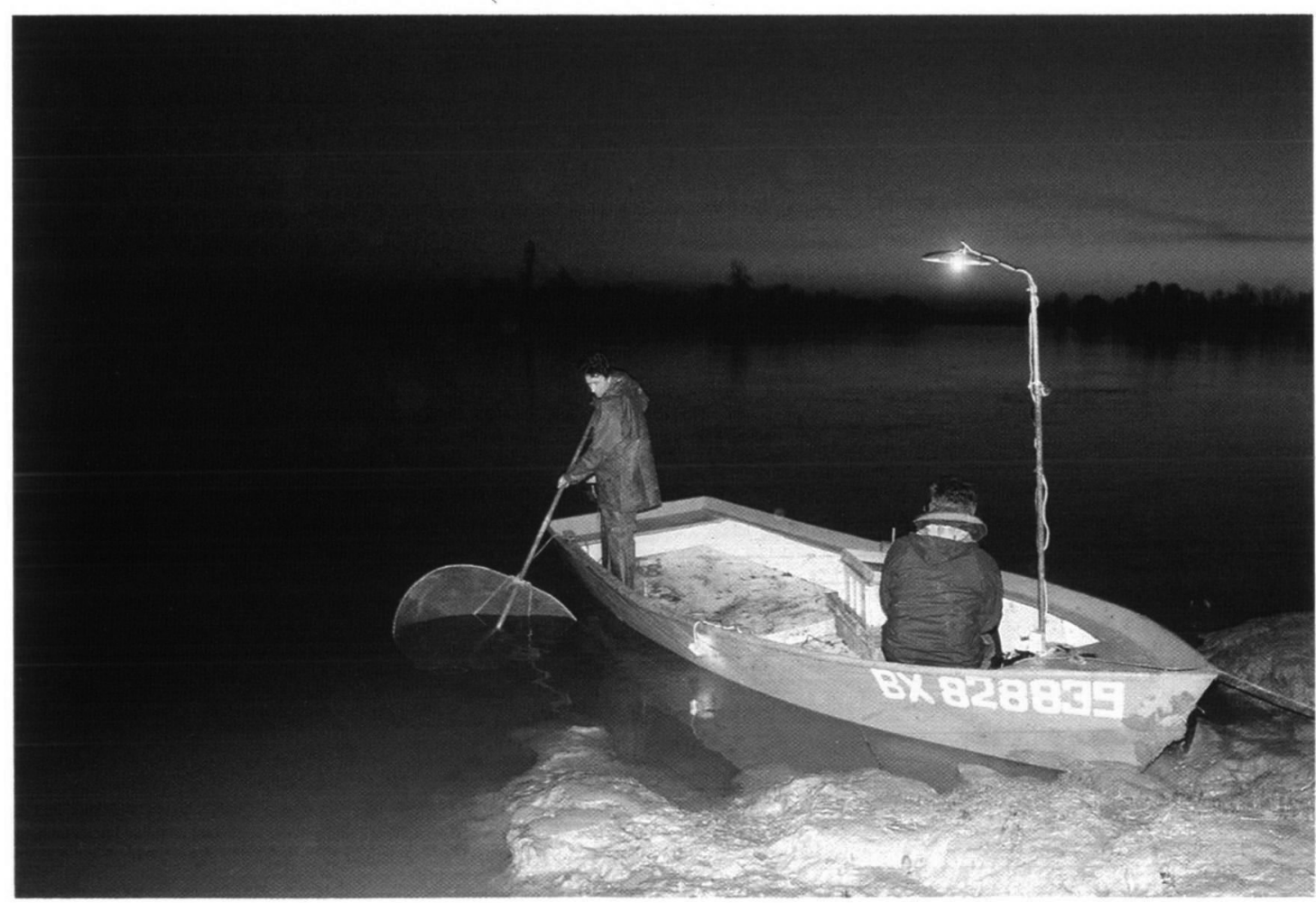

Pêche au tamis à main sur la zone mixte de la Dordogne. 
Les pêcheurs professionnels marins et fluviaux et les amateurs fluviaux licenciés ont été dénombrés par recoupement des listes tenues par les services gestionnaires et les organisations de pêcheurs.

Deux enquêtes (CASTELNAUD et al., 1985 ; CASTELNAUD et BABIN, 1992) consolidées et actualisées par des sondages périodiques (CASTELNAUD et ROCHARD, 1994) ont permis de vérifier la fréquentation des lieux de pêche, d'approcher les stratégies de pêche et d'apprécier le niveau d'assiduité des pêcheurs.

Tout au long de la période 1979-1992, un amateur licencié des zones mixtes de Garonne - Dordogne - Isle a été considéré comme développant un effort égal à celui d'un professionnel pour le métier civelle-tamis afin de tenir compte de la part inconnue liée au braconnage.

Ainsi, l'effort nominal total pour chaque métier a été obtenu par sommation des effectifs de chaque catégorie de pêcheurs.

Les productions ont été calculées à partir de l'effort nominal total et des captures moyennes des pêcheurs coopératifs pour le métier civelle-pibalour sur l'estuaire de la Gironde de 1979 à 1992 et pour le métier civelle-tamis, de façon différenciée sur Garonne et DordogneIsle de 1979 à 1988 et de façon regroupée sur l'ensemble de la zone mixte de 1989 à 1992.

La difficulté d'identifier correctement les sorties de pêche ou marées, du fait de la variabilité des situations de pêche et des temps de pêche, nous a amené à retenir le jour de pêche comme unité d'effort effectif commune aux deux métiers.

Les calculs d'effort effectif en nombre de jours de pêche, de P.U.E. en $\mathrm{kg}$ par jour de pêche ont été effectués de 1979 à 1981 (CANTRELLE, 1981) et de 1983 à 1992: (ROCHARD, 1992 ; CASTELNAUD et ROCHARD, 1994) sur l'estuaire de la Gironde pour le métier civelle-pibalour et sur l'ensemble de la zone mixte Garonne - Dordogne - Isle pour le métier civelle-tamis.

Les trois indicateurs halieutiques C, f, P.U.E., ont été exprimés pour la période 1989 1992 avec un risque statistique de $5 \%$.

\subsection{Bassin de l'Adour}

Sur le bassin de l'Adour, les marins-pêcheurs ont été dénombrés à partir des enregistrements des Affaires Maritimes, du Comité local des Pêches de Bayonne et des fiches de pêche du CIPE.

Depuis 1985 , les déclarations individuelles de captures et d'effort journaliers consignées sur les fiches de pêche du CIPE ont permis d'évaluer les indicateurs halieutiques de l'exploitation de la civelle (GUERAULT et al., 1991 ; PROUZET et al., 1994). Ces indicateurs halieutiques, qui ne concernent qu'une catégorie d'exploitants et une période de temps limitée, ne peuvent être utilisées dans le diagnostic sur l'abondance des civelles. Ils sont par contre pertinents pour l'établissement du bilan sur l'exploitation en 1989.

\section{Conventions, analyse et confrontation des données}

Les pêcheurs amateurs maritimes à pied, les pêcheurs amateurs fluviaux à pied ou en bateau et les braconniers avec ou sans droit de pêche, qui commercialisent leurs prises, seront rassemblés sous l'appellation de pêcheurs non-professionnels. La saison de pêche s'étale de novembre à avril ; par convention, l'année $x$ représentera dans ce texte la saison totale de pêche commençant à la fin de l'année $x-1$.

Les indicateurs halieutiques d'effort et de production de l'année 1989 des bassins de Vilaine, Loire, Gironde, Adour sont utilisés comme base pour dresser l'état de l'exploitation en 1989. Bien que plus anciennes, les estimations des efforts nominaux et des productions issues des enquêtes et des études réalisées dans les autres secteurs concernés par la pêche de la civelle, viennent compléter cet état, car elles sont les seules disponibles.

Les P.U.E., considérées a priori comme des indices d'abondance (en faisant abstraction des incidences des variations de la capturabilité), sont spécifiques d'un métier de pêche donné ; elles ne doivent pas être comparées d'un métier à l'autre, ni d'un bassin à l'autre. 
Pour tenter de statuer sur les tendances de l'abondance des civelles dans chacun des bassins de la Vilaine, de la Loire et de la Gironde, nous interpréterons en parallèle l'évolution des trois indicateurs halieutiques C, f, P.U.E. comme ROCHARD (1992).

\section{RÉSULTATS}

\section{Caractérisation de l'exploitation pour l'année de référence 1989}

La pêche de la civelle se pratique en France uniquement sur les façades de la Manche et de l'Atlantique. On peut distinguer trois grands ensembles : la Manche Est de Dunkerque au Mont Saint-Michel, la Bretagne du Mont Saint-Michel à Vannes, le Golfe de Gascogne de Vannes à Saint-Jean-de-Luz (Tableau II). La localisation géographique des secteurs de pêche est indiquée sur la figure 2.

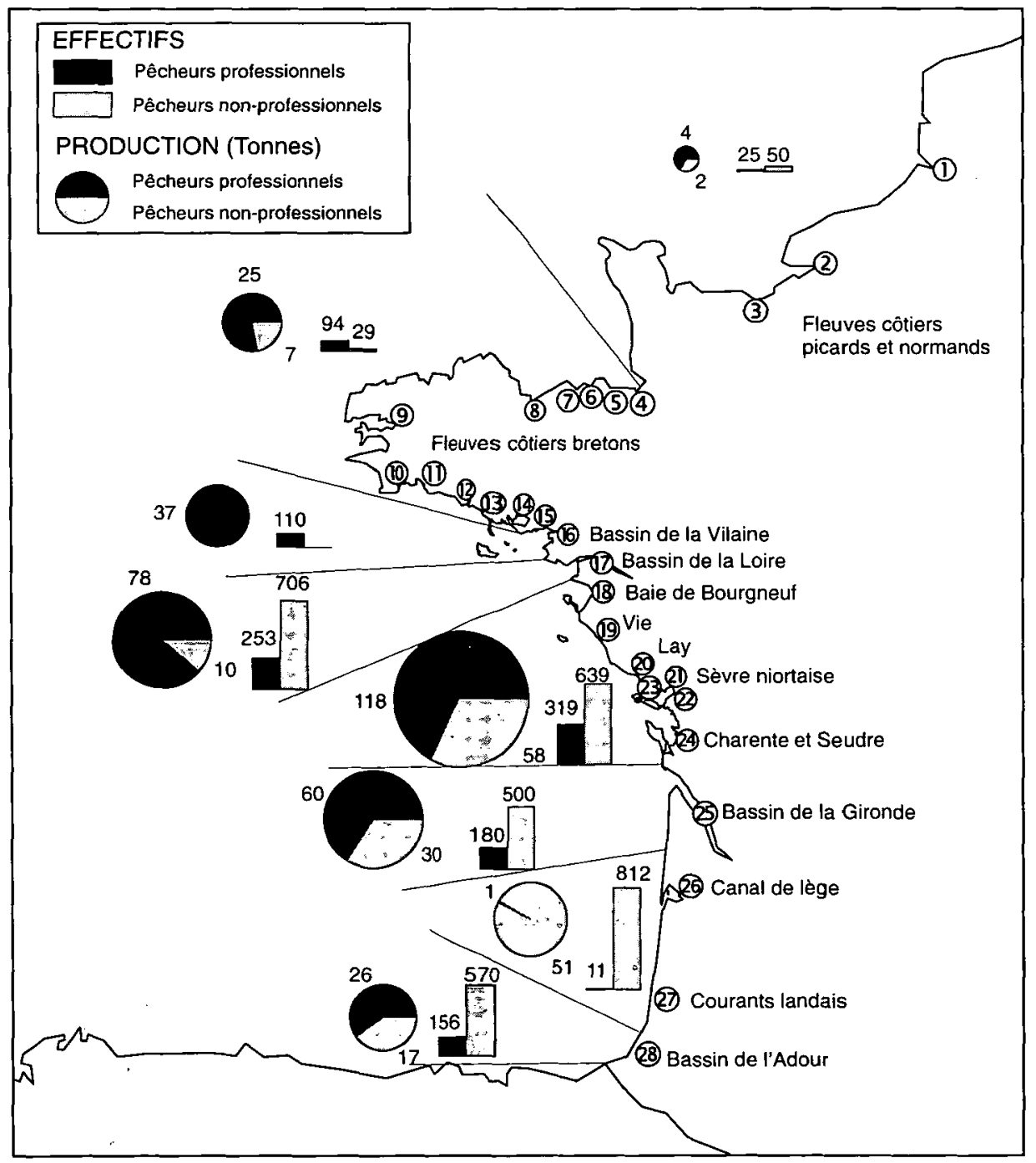

Figure 2 : Secteurs de pêche, effectifs de pêcheurs et production de civelle en France en 1989.

Figure 2 : Fishing areas, number of fishermen and production of civelle in France in 1989. 
Tableau II : L'exploitation de la civelle sur les côtes françaises en 1989 (production en tonnes).

( $\left.{ }^{*}\right)$ amateurs pouvant exercer à la fois en zone maritime et en zone fluviale. 1 à 3 : DESAUNAY $(1987) ; 4$ à 15, 18, 19, 23, 24, 26, 27 et 28' : AUBRUN $(1986 ; 1987)$.

17 et 18 : GUERAULT et al. (1993) ; 20, 21 et 22 : GASCUEL (1987) .

24', 27' et 28' : CASTELNAUD et BABIN (1992).

25 et 25' : SERTIER et al. (1990) ; 28 : PROUZET et al. (1994).

Table II : Exploitation of the civelle in France in 1989 (production in tons).

$\left.{ }^{(}\right)$amateur fishermen who may be on marine and river sections.

1 to 3 : DESAUNAY (1987) ; 4 to $15,18,19,23,24,26,27$ and $28^{\prime}$ : AUBRUN (1986; 1987).

17 and 18 : GUERAULT et al. (1993) ; 20, 21 and 22 : GASCUEL (1987).

24', 27' and 28' : CASTELNAUD and BABIN (1992).

25 and 25' : SERTIER et al. (1990) ; 28 : PROUZET et al. (1994).

\begin{tabular}{|c|c|c|c|c|c|c|c|c|}
\hline \multirow{3}{*}{$\begin{array}{c}\text { CATEGORIES } \\
\text { DE PECHELRS } \\
\text { SECTELIR DE PECHE } \\
\end{array}$} & \multicolumn{3}{|c|}{ PROFESSIONXELS } & \multicolumn{2}{|c|}{ XON PROFESSIOXNELS } & \multirow{3}{*}{ ENGIXs } & \multicolumn{2}{|c|}{ PRODLCTION } \\
\hline & \multicolumn{2}{|c|}{ Naritimes } & \multirow{2}{*}{$\begin{array}{l}\text { Fluviaux } \\
\text { en bateau }\end{array}$} & \multirow{2}{*}{$\begin{array}{l}\text { Maritimes } \\
\text { a pied } \\
\end{array}$} & \multirow{2}{*}{\begin{tabular}{|c|} 
Hluviaux \\
à pied ou en balcau
\end{tabular}} & & \multirow[t]{2}{*}{ Naritime } & \multirow[t]{2}{*}{ Fluviale } \\
\hline & baleau & a pied & & & & & & \\
\hline \multicolumn{9}{|l|}{ MANCH: BST } \\
\hline 1. Sormane & 5 & & & & & \multirow{3}{*}{ I Tamis circulaire } & & \\
\hline 2. Seine, Risle & & $5 \div 10$ & & 20 à 30 & & & $+20 \mathrm{~T}$ & \\
\hline 3- Touques, Dive, Ome, Aure. Vire & & 10 & & 20 & & & & \\
\hline Total $I$ à 3 & $s$ & 15 \& 20 & & $40 \div 50$ & & & $\operatorname{sabT}$ & \\
\hline \multicolumn{9}{|l|}{ BRFTAGNE. } \\
\hline 4. Couesnon & 1 & & & & & $2 \mathrm{~T}$. circulaires $1.20 \mathrm{~m}$ & & \\
\hline 3-St Benoir des $O$. Le Vivier sur $M$ & & 10 & & & & & & \\
\hline 6. Rance & 3 & 20 a 25 & & & & $2 \mathrm{~T}$ rectangulaires $1.20 \mathrm{~m}$ & & \\
\hline 7. Fremur, Arezuenon & 4 & 10 a 15 & & & & $2 \mathrm{~T}$. rectangulaires $1.20 \mathrm{~m}$ & & \\
\hline 8. Gouessant, Gouet & 2 & & & & & $2 \mathrm{~T}$. reciangulaires $120 \mathrm{~m}$ & & \\
\hline 9- Elom, Aulne & 5 & $8 \mathrm{a} 15$ & & $4 \div 7$ & & $1 \mathrm{~T}$. circulaise $1.20 \mathrm{~m}$ & $13 \mathrm{a} 32 \mathrm{~T}$ & \\
\hline 10-Odet, PI-L:Abbe & 7 & & & & & I I circulaise $1.20 \mathrm{~m}$ & & \\
\hline 11. Lata & 1 & & & & 394 & $2 \mathrm{~T}$ circulaires $1.20 \mathrm{~m}$ & & \\
\hline 12-Scorff. Blase & 1 & & & 10 a is & $*$ & $2 \mathrm{~T}$ c circulaires $1.20 \mathrm{~m}$ & & \\
\hline $13-$ riv. dElel & & & & 3 & & . & & \\
\hline 14-Golfe du Marbihan, riv đAuray & & tis & & & & $1 \mathrm{~T}$. circulaire $1.20 \mathrm{~m}$ & & \\
\hline \multicolumn{9}{|l|}{ 15. riv, de Penerf } \\
\hline TOTALA o d $/ 5$ & 24 & 52 a 70 & & 17 a 25 & $3 \dot{44}$ & & $2 J a 32 T$ & \\
\hline \multicolumn{9}{|l|}{ GOLFE DE GASCOGNE } \\
\hline 16. Vilaine & 110 & & & & & 21 . circulaites $1.20 \mathrm{~m}$ & 37 & \\
\hline 17 - Loire mas. ef llux., Sevie Nantaise & 210 & & 4.3 & 500 & 206 & $2 T$. circulaires $1.20 \mathrm{~m}$ & $60 \mathrm{~T}$ & $12 T$ \\
\hline 18 - Baie de Bourgneuf & 3.4. & & & 120 & & $2 \mathrm{~T}$. circulaires $1.20 \mathrm{~m}$ & Sais T & \\
\hline 19. Vie & 13 & & & $10 \mathrm{a} 20$ & & $2 \mathrm{~T}$. rectangulaires $2 \mathrm{~m}$ & $10 \div 15 \mathrm{~T}$ & \\
\hline 20 - Lay & 58 & & & $*$ & 40 a 50 & $2 \mathrm{~T}$. rectangulaires $1.20 \mathrm{~m}$ & $25 \dot{3} .30 \mathrm{~T}$ & $5 \mathrm{a} 10 \mathrm{~T}$ \\
\hline 21 - Setre Nionaise & 70 & & & $*$ & $15 \div 20$ & $2 \mathrm{~T}$ rectangulaires $1.20 \mathrm{~m}$ & 25 \& $30 \mathrm{~T}$ & $4 T$ \\
\hline 22. Canaux Baic de l'Aiguillon & & & & 50 i 60 & & $1 \mathrm{~T}$ cirreulaire $1.20 \mathrm{~m}$ & $15 \mathrm{a} 20 \mathrm{~T}$ & \\
\hline 2.7 - lle de Ré & & $s$ & & 30 & & $1 \mathrm{~T}$. cuculaire $1.20 \mathrm{~m}$ & ST & \\
\hline 24-Charente manitine, Seudre & 137 & & & 300 & & 2 pibalours 4 a $7 \mathrm{~m}$ & .35 i $15 \mathrm{~T}$ & \\
\hline 24-Charense fluxiale & & & 2 & & 39 & 1T. ovale $1.20 \mathrm{~m}$ & & $1.5 \mathrm{~T}$ \\
\hline 25. Gironde, Estuaire maritime & 65 & & & $\div$ & 150 × 200 & 2 pibalours 3 \ $7 \mathrm{~m}^{2}$ & $41 \mathrm{~T}$ & \\
\hline 23' - Garonne, Dordogne, Isle fiuviale & & & 115 & & 300 & IT. ovale $1.200 .50 \mathrm{~m}$ & & $.39 \mathrm{~T}$ \\
\hline 26-Canal de Lë̌re & & & & 30 a so & $*$ & $1 \mathrm{~T}$. orale $1.20 \mathrm{~m}$ & $12 T$ & \\
\hline \multicolumn{4}{|l|}{$\begin{array}{l}\text { 27. Coerants Landais mar. - Mimizan. } \\
\text { Contis, Iluchel, Vicux-Boucau, Capbreton }\end{array}$} & \multirow[t]{2}{*}{700} & 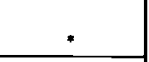 & \multirow[t]{2}{*}{ IT ovale $1.20 \mathrm{~m}$} & \multirow[t]{2}{*}{$40 \mathrm{~T}$} & \\
\hline 27'-Couranis l.anciais fhesiaur & & & 11 & & 62 & & & \\
\hline 28 - Adour. Nive, Bidouse, Luv. Gares & 65 & & & & & \multirow{3}{*}{ IT. ovale $1.20 \mathrm{~m}$} & $\underline{9 T}$ & \\
\hline $28^{\circ}$ - Adour et amlients maritimes & & & & 200 & 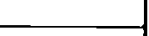 & & 5 a $7 \mathrm{~T}$ & \\
\hline $28^{\circ}$ - Adour et afllucnis flusiaux & & & 91 & & .370 & & & $16.5 \mathrm{~T}$ \\
\hline TOLAI. 16 a $28^{\prime \prime}$ & 762 & 5 & 262 & $19.40+1980$ & $1182 \& 1247$ & & 333 a $372 T$ & $78 \dot{a} 83 \mathrm{~T}$ \\
\hline TOTAL GENERAL & 791 & 72195 & 262 & $1997+2055$ & $1185+12591$ & & $360+410 \mathrm{~T}$ & $78 \gg 83 \mathrm{~T}$ \\
\hline
\end{tabular}


Sur le premier ensemble «Manche Est», les seules données disponibles concernent l'année 1986 (DESAUNAY, 1987).

5 marins-pêcheurs en bateau, de 15 à 20 marins-pêcheurs à pied et 40 à 50 amateurs maritimes à pied exerçaient sur la Somme, la Seine et la Risle et les rivières du Calvados ; ces pêcheurs utilisaient un tamis circulaire de 1,20 m. La production était estimée à 4 à 6 tonnes.

Sur le deuxième ensemble "Bretagne», les seules données disponibles concernent aussi l'année 1986 (AUBRUN, 1986). 24 marins-pêcheurs exerçaient en bateau équipé de 1 ou 2 tamis circulaires ou rectangulaires, sur le Couesnon, la Rance, le Frémur et l'Arguenon, le Gouet, l'Elorn, l'Aulne, l'Odet et la rivière Pont-l'Abbé, le Blavet. 50 à 70 marins-pêcheurs y pêchaient à pied, ainsi que 20 à 30 amateurs maritimes dont une dizaine se retrouvent aussi en zone sous réglementation fluviale du Blavet. 23 à 32 tonnes de civelles ont été débarquées en Bretagne (Tableau II).

Sur le troisième ensemble «Golfe de Gascogne», les données les plus récentes pour la Baie de Bourgneuf, la Vie, les chenaux de l'lle de Ré, la Charente, la Seudre, le canal de Lège, datent aussi de 1986 (AUBRUN, 1987). Pour le Lay, la Sèvre Niortaise et les canaux de la baie d'Aiguillon, il faut remonter à 1983 (GASCUEL, 1987).

Environ 170 marins-pêcheurs en bateau poussaient 2 tamis circulaires dans la baie de Bourgneuf, la Vie, le Lay, la Sèvre Niortaise ; 137 marins pêcheurs en bateau poussaient 2 pibalours de 4 à $7 \mathrm{~m}^{2}$ sur la Charente et la Seudre; 5 à 6 marins-pêcheurs exerçaient à pied dans les chenaux de l'lle de Ré. 550 à 600 amateurs à pied, surtout maritimes, pêchaient au tamis à main dans ces secteurs. On trouvait uniquement des pêcheurs de cette catégorie sur le canal de Lège relié au bassin d'Arcachon, au nombre de 50 environ. L'ensemble de ces pêcheurs totalisait une production allant de 135 à 173 tonnes.

Des pêcheurs fluviaux capturant la civelle ont été répertoriés uniquement sur la Charente où CASTELNAUD et BABIN (1992) comptaient, en 1989, 2 professionnels et 39 amateurs licenciés. D'après BABIN (1993), ils avaient produit environ 1,5 tonne en 1989 (Tableau II).

Pour les autres secteurs du Golfe de Gascogne qui sont, avec le secteur CharenteSeudre, les plus importants pour la pêche de la civelle, nous possédons des données d'effort et de production pour l'année 1989.

Sur le bassin de la Vilaine et sur le bassin de la Loire, les pêcheurs professionnels capturaient la civelle avec 2 tamis circulaires (diamètre $1,20 \mathrm{~m}$, profondeur : 1,30 m) fixés de chaque côté de l'embarcation et tractés à une vitesse de l'ordre de 3 ou 4 noeuds. Les non-professionnels maritimes et fluviaux pêchaient la civelle à partir des berges avec un tamis monté sur balancine ou emmanché suivant la configuration des lieux de pêche (appontements, étiers). Le diamètre du tamis est généralement de l'ordre de 0,50 $\mathrm{m}$.

Sur le bassin de la Vilaine, le barrage d'Arzal limite l'exercice de la pêche à l'aval de l'estuaire sur une longueur maximale de $5 \mathrm{~km}$. Elle est très réglementée et exclut désormais toute pêche amateur. La production des 110 marins-pêcheurs dénombrés en 1989 a été estimée à 37 tonnes (30 tonnes selon les statistiques officielles) d'après GUERAULT et al., 1993 (Tableau III).

Sur le bassin de la Loire, la pêche se déroule sur près de $70 \mathrm{~km}$, dans un secteur formé de l'estuaire maritime de la Loire, de la zone mixte de la Loire et de la Sèvre Nantaise.

En 1989, d'après GUERAULT et al. (1993) on comptait 210 marins-pêcheurs dont 112 d'entre eux avaient acquitté des taxes leur donnant accès à tout ou partie des zones mixtes alors que 43 professionnels fluviaux (40 d'après CASTELNAUD et BABIN, 1992) exploitaient la civelle dans les zones mixtes sous réglementation fluviale (Tableau III).

206 amateurs fluviaux licenciés ont également participé à l'exploitation de la civelle dans les zones mixtes ainsi que 500 amateurs maritimes environ (évaluation à partir d'enquêtes de terrain partielles). La production des marins-pêcheurs et des professionnels fluviaux a été estimée respectivement à 66 et 12 tonnes (61 et 9 tonnes d'après les statistiques des Affaires Maritimes et de la DDAF). D'après BABIN (1993), les professionnels fluviaux ont capturé 15 tonnes en 1989. La production des nonprofessionnels n'a pu être estimée (GUERAULT et al., 1993). 
Tableau III : La pêche de la civelle dans les quatre principaux bassins de la façade atlantique en 1989 (Prix moyen : 400 F/kg) ; MP : marins-pêcheurs ; PF : professionnels fluviaux.

Table III : The civelle fishery in the four main basins emerging in the Atlantic ocean (mean price : 400 F.F./kg) ; MP : marine professional fishermen ; PF : river professional fishermen.

\begin{tabular}{|c|c|c|c|c|c|c|}
\hline \multirow{3}{*}{ Métier } & \multirow{3}{*}{ Secteur } & \multicolumn{2}{|c|}{ Effectifs de pezcheurs } & \multicolumn{2}{|c|}{ Production $(\mathrm{Kg})$} & \multirow{3}{*}{ BASSIN DELA VILAINI } \\
\hline & & Population & Echantillon & Captures moyennes & Captures totales & \\
\hline & & MP & MP & MP & MP & \\
\hline $\begin{array}{l}\text { Pèche professionnelle } \\
\text { au tamis }\end{array}$ & $\begin{array}{c}\text { Vilaine } \\
\text { (Zone maritime) }\end{array}$ & 110 & 49 & 334 & 36700 & $\begin{array}{l}\text { Production : } 36,7 \text { tonnes } \\
\text { Valeur : } 14,7 \mathrm{MF}\end{array}$ \\
\hline
\end{tabular}

\begin{tabular}{|c|c|c|c|c|c|c|c|c|c|c|}
\hline \multirow{3}{*}{ Mettier } & \multirow{3}{*}{ Secteur } & \multicolumn{4}{|c|}{ Effectifs de pêcheurs } & \multicolumn{4}{|c|}{ Production (Kg) } & \multirow{3}{*}{ BASSIN DE LA LOLRE } \\
\hline & & \multicolumn{2}{|c|}{ Population } & \multicolumn{2}{|c|}{ Echantillon } & \multicolumn{2}{|c|}{ Captures moyennes } & \multicolumn{2}{|c|}{ Captures totales } & \\
\hline & & MP & PF & MP & PF & MP & PF & MP & $\mathbf{P F}$ & \\
\hline $\begin{array}{l}\text { Pesche } \\
\text { professionnelle }\end{array}$ & $\begin{array}{c}\text { Loire (Zone maritime) } \\
\text { Loire et Stvre Nantaise } \\
\text { (Zones mixtes) }\end{array}$ & 210 & & 65 & & 312 & & 65500 & & Production : 77.8 tonnes \\
\hline $\begin{array}{l}\text { au } \\
\text { tamis }\end{array}$ & $\begin{array}{l}\text { Loire et Stvre Nantaise } \\
\text { (Zones mixtes) }\end{array}$ & & 43 & & 26 & & 286 & & 12300 & Valeur : $31.1 \mathrm{MF}$ \\
\hline
\end{tabular}

\begin{tabular}{|c|c|c|c|c|c|c|c|}
\hline \multirow[b]{2}{*}{ Métier } & \multirow[b]{2}{*}{ Secteur } & \multicolumn{2}{|c|}{ Effectifs de pezcheurs } & \multicolumn{2}{|c|}{ Production (Kg) } & \multirow{2}{*}{\multicolumn{2}{|c|}{$\begin{array}{l}\text { BASSIN GLRONDE- } \\
\text { GARONNE-DORDOGNE }\end{array}$}} \\
\hline & & Population & Echantillon & $\begin{array}{l}\text { Captures } \\
\text { moyennes }\end{array}$ & $\begin{array}{c}\text { Captures } \\
\text { totales }\end{array}$ & & \\
\hline $\begin{array}{c}\text { Pèche } \\
\text { professionnelle } \\
\text { Pibalour } \\
\end{array}$ & $\begin{array}{c}\text { Gironde } \\
\text { (Zone maritime) }\end{array}$ & 65 & 7 & 630 & 41000 & $\begin{array}{l}\text { Production : } 41 \text { tonnes } \\
\text { Valeur : } 16.4 \mathrm{MF}\end{array}$ & \multirow{3}{*}{$\begin{array}{l}\text { Production : } 80.1 \text { tonnes } \\
\text { Valeur : } 32 \mathrm{MF}\end{array}$} \\
\hline $\begin{array}{c}\text { Peche } \\
\text { professionnelle } \\
\text { aut tamis }\end{array}$ & $\begin{array}{c}\text { Garonne, Dordogne } \\
\text { et Isle } \\
\text { (Zones mixtes) }\end{array}$ & 115 & \multirow[t]{2}{*}{14} & 161.5 & 18600 & $\begin{array}{l}\text { Production : } 18.6 \text { tonnes } \\
\text { Valeur : } 7.4 \mathrm{MF}\end{array}$ & \\
\hline $\begin{array}{l}\text { Pèche non } \\
\text { professionnelle } \\
\text { au tamis }\end{array}$ & $\begin{array}{c}\text { Garonne, Dordogne } \\
\text { et Isle } \\
\text { (Zones mixtes) }\end{array}$ & 127 & & 161.5 & 20500 & $\begin{array}{l}\text { Production : } 20.5 \text { tonnes } \\
\text { Vateur : } 8.2 \mathrm{MF}\end{array}$ & \\
\hline
\end{tabular}

\begin{tabular}{|c|c|c|c|c|c|c|}
\hline \multirow{3}{*}{ Metier } & \multirow{3}{*}{ Secteur } & \multicolumn{2}{|c|}{ Effectifs de pêcheurs } & \multicolumn{2}{|c|}{ Production (Kg) } & \multirow{3}{*}{ BASSIN DE L'ADOUR } \\
\hline & & Population & Echantillon & Captures moyennes & Captures totales & \\
\hline & & MP & MP & MP & MP & \\
\hline $\begin{array}{c}\text { Pēche } \\
\text { professionnelle } \\
\text { au tamis }\end{array}$ & $\begin{array}{l}\text { Adour (Zone maritime) } \\
\text { Adour et Gaves retunis } \\
\text { (Zones mixtes) }\end{array}$ & 65 & 40 & 139 & 9000 & $\begin{array}{l}\text { Production : } 9 \text { tonnes } \\
\text { Valeur : } 3.6 \mathrm{MF}\end{array}$ \\
\hline
\end{tabular}

En 1989, sur le bassin de la Gironde, long de 150 km, 65 marins-pêcheurs poussaient deux pibalours de 3 à $7 \mathrm{~m}^{2}$ dans l'estuaire maritime (Tableau III) ; 115 professionnels dont 109 fluviaux et 6 marins exerçaient au tamis de $1,20 \mathrm{~m}$ de diamètre manoeuvré à la main depuis un bateau dans les zones mixtes sous réglementation fluviale de Garonne-Dordogne et Isle, concurremment avec 127 amateurs fluviaux licenciés (SERTIER et al., 1990). Les amateurs sont à pied ou en bateau; ils utilisent des tamis 
amateurs de $50 \mathrm{~cm}$ de diamètre ou des tamis de professionnels. 125 pêcheurs aux lignes avaient un droit de pêche au tamis amateur en 1989 et exerçaient sur les chenaux bordant l'estuaire et appartenant au Domaine Privé.

Un nombre inconnu, mais important, de braconniers exerce pendant les périodes réglementaires mais aussi le week-end lors de la relève hebdomadaire et après la date de fermeture dans les zones mixtes des fleuves et dans les chenaux bordant l'estuaire sur le Domaine Public et sur le Domaine Privé. Compte tenu des effectifs de pêcheurs amateurs licenciés et des estimations des effectifs de non-professionnels en action de pêche proposées par la Garderie, nous avons retenu pour l'année 1989 le nombre de 300 pêcheurs non-professionnels à pied ou en bateau sur la zone mixte et de 150 à 200 en bordure d'estuaire (Tableau II).

La production des marins-pêcheurs au pibalour dans la Gironde a été estimée à 41 tonnes et celle des pêcheurs fluviaux, professionnels et non-professionnels au tamis, à 39 tonnes. La part des pêcheurs non-professionnels correspondait à $52 \%$ de la production totale des zones mixtes et à $26 \%$ de la production totale du bassin.

Sur le bassin de l'Adour, PROUZET et al. (1994) comptaient en 1989 environ 65 marins-pêcheurs capturant au tamis de $1,20 \mathrm{~m}$ de diamètre, manoeuvré à la main depuis un bateau, 9 tonnes de civelles en 1989 (Tableau III). AUBRUN (1986) avançait pour 1986 un chiffre d'environ 200 amateurs maritimes à pied produisant de 5 à 7 tonnes de civelles (Tableau II).

En zone sous réglementation fluviale, exerçaient 91 professionnels fluviaux et 370 amateurs et cotisants Solidarité (5) en 1989 d'après CASTELNAUD et BABIN, (1992), chiffres proches de ceux de AUBRUN (1986). La production des professionnels a été estimée par BABIN (1993) à 16,5 tonnes en 1989 (Tableau II).

Sur les courants landais, AUBRUN (1986), estimait à 700 les pêcheurs amateurs à pied, maritimes et fluviaux ; CASTELNAUD et BABIN (1992) dénombraient 11 professionnels et 62 amateurs licenciés en zone fluviale. La production donnée par AUBRUN (1986), pour l'année 1986 est de 40 tonnes. (Tableau II).

Au bilan, compte tenu des inconnues et des approximations, nous proposons de retenir, sur la base des résultats synthétiques du tableau II, les chiffres arrondis suivants, pour l'année de référence 1989 :

\section{PÊCHEURS}

- 100 marins-pêcheurs professionnels à pied dont l'essentiel exerce en Bretagne ;

- 800 marins-pêcheurs professionnels en bateau dont l'essentiel exerce dans les estuaires du Golfe de Gascogne (auxquels correspondent 967 licences CIPE attribuées en 1989);

- 260 pêcheurs professionnels en eau douce qui pêchent dans les zones mixtes des grands fleuves de la côte atlantique ;

-2000 pêcheurs maritimes non-professionnels à pied dont l'essentiel se trouve sur les estuaires et le littoral du Golfe de Gascogne et dont une partie pêche aussi en zone sous réglementation fluviale dans les Landes notamment ;

- 1200 pêcheurs fluviaux non-professionnels dans les mêmes zones mixtes dont 2 à 300 exercent en bateau surtout dans les zones mixtes Garonne - Dordogne - Isle (auxquels correspondent 414 taxes piscicoles civelles professionnelles et 606 taxes piscicoles civelles amateurs délivrées en 1989).

\section{PRODUCTION}

- 410 tonnes débarquées par les pêcheurs maritimes dont les trois quarts (300 tonnes) par les marins-pêcheurs professionnels ;

(5) Cotisant Solidarité : pêcheur licencé civelle non-professionnel, commercialisant ses prises et bénéficiant d'un statut particulier dans les Landes. 
- 80 tonnes capturées par les pêcheurs fluviaux auxquelles on adjoindra arbitrairement la production non comptabilisée au Tableau 2 des non-professionnels marins et fluviaux de Loire (10 t.), d'une partie des non-professionnels de Gironde (10 t.) et de l'Adour (10 t.), ce qui conduit à un total de 110 tonnes environ ; 40 à $45 \%$ sont issus de la pêche professionnelle.

Ainsi, 4360 pêcheurs dont 1160 pêcheurs professionnels (27\%) et 3200 nonprofessionnels $(73 \%)$ ont débarqué 520 tonnes de civelles en 1989, réalisant un chiffre d'affaires de plus de 200 millions de francs. Dans ces estimations, la part des nonprofessionnels représente $33 \%$ des apports, soit 170 tonnes et 68 millions de francs.

La figure 2 ventile les chiffres du tableau II selon deux groupes de pêcheurs, professionnels et non-professionnels, dans 8 grands secteurs de pêche.

Le prix moyen de $400 \mathrm{~F} / \mathrm{kg}$ atteint en 1989 était un prix plafond qui a eu tendance à baisser depuis. II avait triplé en francs courants depuis 1983 et doublé la dernière année ; en francs constants cela fait une augmentation de 2,5 entre 1983 et 1989.

\section{2. Évolution inter-annuelle de l'abondance des flux de civelle}

Les résultats ont donné lieu à une interprétation à deux échelles de temps (moyen et court terme).

\subsection{Bassin de la Vilaine et de la Loire}

\subsection{1. Évolution inter-annuelle de l'abondance à moyen terme (1977-1990)}

\section{Variation des productions globales à moyen terme}

Les captures étaient de l'ordre de 500 à 700 tonnes de 1977 à 1980 en Loire et de 100 tonnes en Vilaine pour la même période. Elles n'étaient plus respectivement que de l'ordre d'une centaine et d'une trentaine de tonnes dans chaque estuaire à la fin des années 80. La diminution des productions s'est amorcée en 1981 en Loire et en 1980 en Vilaine (Tableau IV). Elle est d'autant plus sensible que la fin des années 70 correspondait vraisemblablement à des années records, en particulier en Loire (GUERAULT et al., 1993).

\section{Variation des indices établis à partir de sélection et de lots témoins de pêcheurs}

Les deux séries de prises par unité d'effort obtenues à partir de la sélection et du lot témoin de pêcheurs étaient du même ordre dans chaque estuaire et schématisent une évolution pratiquement synchrone dans les deux estuaires (Tableau IV). Les rapports entre les valeurs moyennes maximales des saisons $1977,1978,1979(28,3 \mathrm{~kg} / \mathrm{sortie}$ en Loire ; $15,4 \mathrm{~kg} / \mathrm{sortie}$ en Vilaine) et les valeurs minimales observées au cours des trois dernières saisons successives 1988, 1989, 1990 (5,9 kg/sortie en Loire ; 4,2 kg/sortie en Vilaine) témoignent d'une division par 4,8 et 3,6 des rendements par sortie de la sélection de pêcheurs en Loire et en Vilaine à l'échelle de la dernière décennie.

Les moyennes individuelles de production et d'effort effectif du lot témoin de pêcheurs montrent en outre, en admettant une relative stabilité de l'effort total déployé sur la pêcherie au cours de deux années successives (1979 et 1980), que le maintien de la production à un niveau élevé en 1980 en Loire n'a été dû qu'à une augmentation brutale de l'effort de pêche effectif (nombre de sorties) d'après GUERAULT et al. $(1989 ; 1991)$.

Malgré le manque d'informations sur l'évolution de l'effort de pêche total et les conditions de capturabilité, la juxtaposition de l'évolution des statistiques officielles de production en termes de tendance et des prises par unité d'effort, laisse penser qu'une réelle réduction d'abondance s'est amorcée simultanément au cours de la saison 1980 dans les deux estuaires et s'est poursuivie depuis.

\subsection{2. Évolution inter-annuelle de l'abondance à court terme (1985 à 1990)}

Sur le bassin de la Vilaine, l'effort effectif s'est accru de 1985 à 1990 à cause de l'augmentation conjointe de l'effort nominal et du nombre de sorties individuelles. La 
Tableau IV : L'exploitation de la civelle dans les bassins de la Vilaine et de la Loire de 1977 à 1990.

Statistiques de production - Indices d'effort, de production et P.U.E. (1977 à 1990) - Indicateurs halieutiques (1985 à 1990).

Table IV : The civelle fishery in the Vilaine and Loire basins from 1977 to 1990.

Production statistics - Effort indices, production indices and P.U.E. (1977 1990) - Fishing indicators (1985 - 1990).

\begin{tabular}{|c|c|c|c|c|c|c|c|c|c|c|c|c|c|c|c|c|}
\hline \multicolumn{2}{|c|}{ BASSIN DE LA VILAINE } & ANNEES & 1977 & 1978 & 1979 & 1980 & 1981 & 1982 & 1983 & 1984 & 1985 & 1986 & 1987 & 1988 & 1989 & 1990 \\
\hline $\begin{array}{c}\text { Donsees } \\
\text { OfficiellLes }\end{array}$ & \multicolumn{2}{|c|}{$\begin{array}{c}\text { STATISHOUES DE CAPTURES (TONNES) } \\
\text { (MARINS-PECHEURS) }\end{array}$} & 32 & 105 & 209 & 95 & 57 & 98 & 69 & 36 & 32 & 48 & 32 & 39 & 30 & 31 \\
\hline \multirow{6}{*}{ Indices } & \multirow[t]{2}{*}{ Selection } & Nombre de pecheurs & 49 & 48 & 52 & 38 & 64 & 48 & 54 & 48 & 40 & 49 & 38 & 59 & 54 & 65 \\
\hline & & P.U.E. (Kg/ sortie) & 11.4 & 19.5 & 15.2 & 8.8 & 5.7 & 8.5 & 5.1 & 6.7 & 6.2 & 6.4 & 5 & 5.2 & 3.9 & 3.6 \\
\hline & \multirow{4}{*}{ Lot temoin } & Nombre de pécheurs & $\therefore$ & . & 4 & 4 & 4 & 4 & 4 & 4 & 4 & 4 & 4 & 4 & 4 & 4 \\
\hline & & $\begin{array}{l}\text { Effor effectif moyen } \\
\text { (Nombre de sorties) }\end{array}$ & & - & 125 & 134 & 138 & 123 & 129 & 90 & 86 & 105 & 116 & 126 & 123 & 86 \\
\hline & & Production moyenne $\left(\mathrm{K}_{g}\right)$ & $=$ & . & 2146 & 1094 & 760 & 846 & 681 & 617 & 452 & 575 & 547 & 543 & 458 & 282 \\
\hline & & P.U.E. $(\mathrm{Kg} /$ sonie) & - & . & 17.2 & 8.1 & 5.5 & 6.9 & 5.3 & 6.8 & 5.3 & 5.5 & 4.7 & 4.3 & 3.7 & 3.3 \\
\hline \multirow{3}{*}{$\begin{array}{l}\text { INDICATEURS } \\
\text { HALIEUTIQUES } \\
\text { OE }\end{array}$} & Echantillon & Nombre de pecheurs & & & & & & & & & 28 & 30 & 33 & $\$ 2$ & 49 & 48 \\
\hline & \multirow{4}{*}{ Population } & Nombre de pecheurs & & & & & & & & & 100 & 91 & 105 & 106 & III & 146 \\
\hline & & $\begin{array}{l}\text { Effor effectif total } \\
\text { (Nombre de sorties) }\end{array}$ & & & & & & & & & 6921 & 7896 & 3955 & 8990 & 9498 & 9940 \\
\hline \multirow[t]{2}{*}{ L'EXPLOITATION } & & Production totale (Tonnes) & & & & & & & & & 41.1 & 52.6 & 41.2 & 46.6 & 36.7 & 35.9 \\
\hline & & P.U.E. (Kg / sortie) & & & & & & & & & 5.9 & 6.7 & 5.2 & 5.2 & 3.9 & 3.6 \\
\hline
\end{tabular}

\begin{tabular}{|c|c|c|c|c|c|c|c|c|c|c|c|c|c|c|c|c|}
\hline \multicolumn{2}{|c|}{ BASSIN DE LA LOIRE } & ANNEES & 1977 & 1978 & 1979 & 1980 & 1981 & 1982 & 1983 & 1984 & 1985 & 1986 & 1987 & 1988 & 1989 & 1990 \\
\hline $\begin{array}{c}\text { DONNEES } \\
\text { OFFICIELLLES }\end{array}$ & \multicolumn{2}{|c|}{ 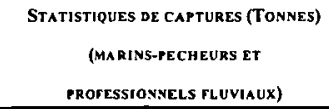 } & 677 & 526 & 641 & 525 & 303 & 274 & 260 & 182 & 168 & 147 & 107 & 149 & 70 & 91 \\
\hline \multirow{6}{*}{ INDICEs } & \multirow[t]{2}{*}{ Selection } & Nombre de pêcheurs & 38 & 48 & 49 & 15 & 15 & 13 & 69 & 68 & 53 & 38 & 43 & 48 & 38 & 45 \\
\hline & & P.U.E. (Kg / sortie) & 26.8 & 31.6 & 26.4 & 20.8 & 16.1 & 13.6 & 9.8 & 9 & 8.2 & 8 & 8.3 & 9 & 4.3 & 4.4 \\
\hline & \multirow{4}{*}{ Lot témoin } & Nombre de pêcheurs & 9 & $9^{\circ}$ & 9 & 9 & 9 & 9 & 9 & 9 & 9 & 9 & 9 & 8 & 9 & 9 \\
\hline & & $\begin{array}{l}\text { Effor effectif moyen } \\
\text { (Nombre de sornies) }\end{array}$ & 85 & 76 & 74 & 108 & 104 & 107 & 107 & 90 & 88 & 79 & 96 & 111 & 91 & 95 \\
\hline & & Production moyenne $(\mathrm{Kg})$ & 2704 & 2819 & 2398 & 2385 & 1664 & 1470 & 1292 & 1022 & 884 & 737 & 847 & 1088 & 406 & 426 \\
\hline & & P.U.E. (Kg/sonie) & 31.8 & 36.9 & 32.3 & 22.1 & 16.1 & 13.7 & 12.1 & 11.3 & 10.1 & 9.3 & 8.8 & 9.8 & 4.5 & 4.5 \\
\hline \multirow{3}{*}{$\begin{array}{l}\text { INDicateurs } \\
\text { MALIEUTIQUES } \\
\text { DE }\end{array}$} & Echantillon & Nombre de pecheurs & & & & & & & & & 88 & 113 & 102 & 96 & 91 & 92 \\
\hline & \multirow{4}{*}{ Population } & Nombre de pecheurs & & & & & & & & & 285 & 268 & 265 & 264 & 253 & 244 \\
\hline & & $\begin{array}{l}\text { Effort effectif total } \\
\text { (Nombre de sorties) }\end{array}$ & & & & & & & & & 16224 & 13207 & 15481 & 20683 & 17457 & 17626 \\
\hline \multirow[t]{2}{*}{ L'EXPLOLTATION } & & Production toatale (Tornes) & & & & & & & & & 144.9 & 113.21 & 131 & 164.7 & 77.8 & 80.4 \\
\hline & & P.U.E. ( $\mathrm{Kg} /$ sortie $)$ & & & & & & & & & 8.9 & 8.6 & 8.5 & 8 & 4.5 & 4.6 \\
\hline
\end{tabular}


production a varié de 41 tonnes à 53 tonnes de 1985 à 1988 (Tableau IV). Elle n'était plus que de l'ordre de 36 tonnes en 1989 et 1990 mais doit être considérée comme relativement stable compte tenu de l'intervalle de confiance des estimations. La prise par unité d'effort a décliné par contre légèrement en 1989 et 1990 . L'évolution relative des indicateurs halieutiques (fig. 3) suggère une légère diminution de l'abondance des flux annuels de civelle en 1989 et 1990 malgré les intervalles de confiance (GUERAULT et al., 1993).
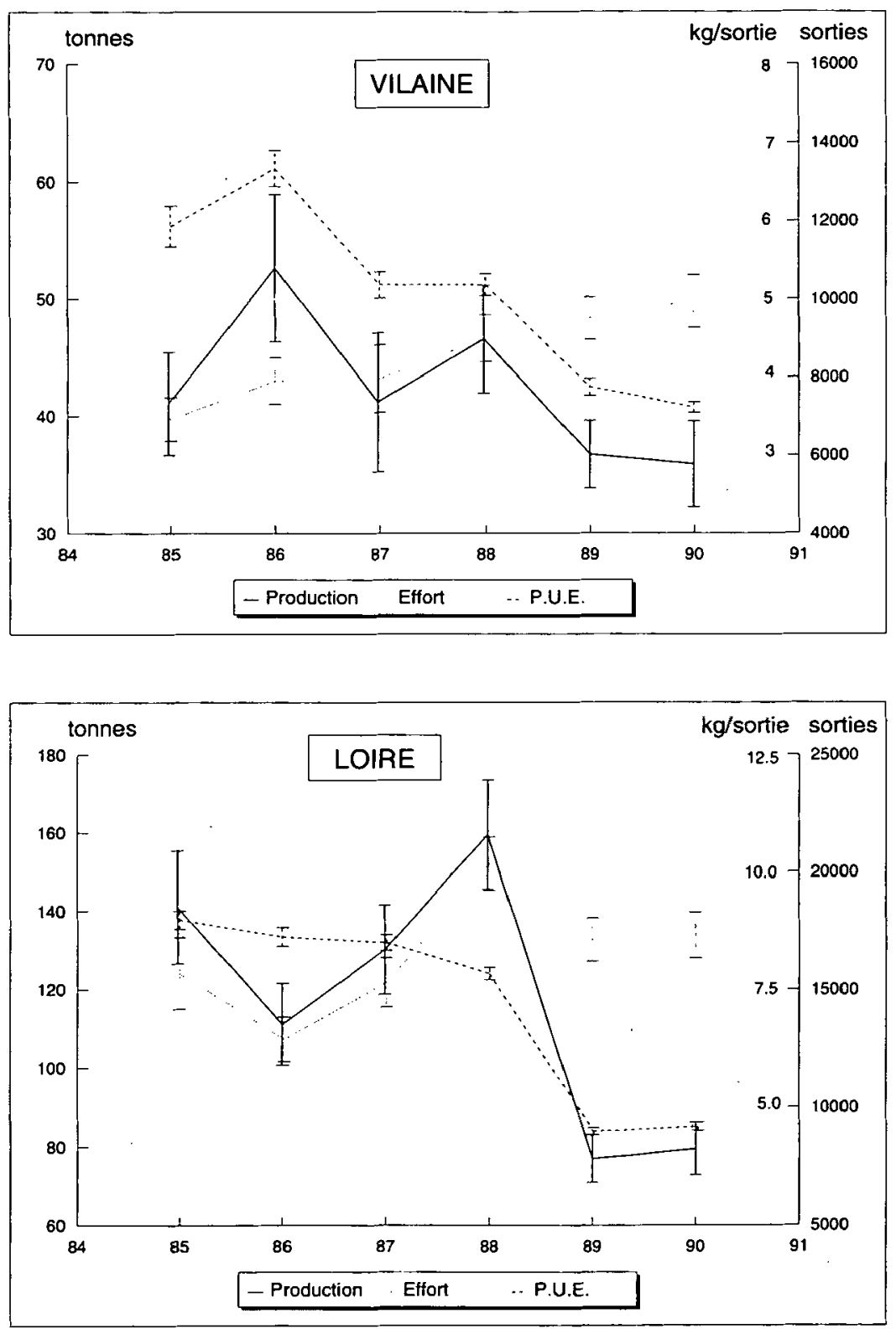

Figure 3 : Évolution des indicateurs halieutiques dans les bassins de la Vilaine et de la Loire de 1985 à 1990 ( I = intervalle de confiance des estimations, sécurité $95 \%$ ).

Figure 3 : Evolution of the fishing indicators in the Vilaine and Loire basins from 1985 to 1990 . ( 1 = confidence interval, security $95 \%$ ). 
Sur le bassin de la Loire, l'analyse de l'évolution relative des trois indicateurs halieutiques à l'échelle de la pêcherie professionnelle (fig. 3) montre une relative stabilité de l'effort effectif alors que l'effort nominal décline, ainsi qu'une diminution drastique de la production et de la prise par unité d'effort en 1989 et 1990 (Tableau IV). Malgré la brièveté de la série de données, l'évolution conjointe de C, f, P.U.E. suggère que les flux saisonniers de civelle sont restés à peu près stables de 1985 à 1988 mais ont chuté au cours des deux dernières saisons de pêche étudiées.

\subsection{Bassin de la Gironde}

\subsubsection{Evolution inter-annuelle de l'abondance à moyen terme (1979 - 1992)}

Nous avons distingué trois périodes concomitantes d'évolution des indicateurs halieutiques pour les deux métiers pratiqués dans ce bassin.

De 1979 à 1981, les captures totales étaient élevées pour les deux métiers, en moyenne 63 tonnes pour le métier civelle-pibalour et 278 tonnes pour le métier civelletamis. Les efforts nominaux et effectifs totaux étaient stables et les P.U.E. élevées, en moyenne $21 \mathrm{~kg} / \mathrm{j}$ pour le métier civelle-pibalour et $20 \mathrm{~kg} / \mathrm{j}$ pour le métier civelle-tamis (Tableau V).

Entre la première période 1979 - 1981 et la seconde période $1982-1988$, les captures totales ont été divisées par 2 en moyenne pour le métier civelle-pibalour et par 7 pour le métier civelle-tamis ; corrélativement, les P.U.E. ont décliné progressivement de moitié pour le métier civelle-pibalour alors que l'effort nominal et l'effort effectif total sont restés globalement stables ; les P.U.E. pour le métier civelle-tamis ont chuté dans la même proportion que les captures totales, passant à une moyenne de $3 \mathrm{~kg} / \mathrm{j}$ tandis que l'effort nominal et l'effort effectif ont diminué graduellement et de façon proportionnelle.

Tableau V : L'exploitation de la civelle dans les bassins de la Gironde de 1979 à 1992. Table V : The civelle fishery in the Gironde basin from 1979 to 1992.

\begin{tabular}{|c|c|c|c|c|c|c|c|c|c|c|c|c|c|c|c|c|}
\hline \multicolumn{2}{|c|}{ BASSIN DE LA GIRONDE } & ANNEES & 1979 & 1980 & 1981 & 1982 & 1983 & 1984 & 1985 & 1986 & 1987 & 1988 & 1989 & 1990 & 1991 & 1992 \\
\hline \multirow{5}{*}{$\begin{array}{l}\text { INDICATEURS } \\
\text { HALIEUTTOUES } \\
\text { DE } \\
\text { L'EXPLOITATION }\end{array}$} & Echantillon & Nombre de pêcheurs & 8 & 8 & 4 & 4 & 4 & 5 & 3 & 7 & 6 & 10 & 7 & 11 & 13 & 12 \\
\hline & \multirow{4}{*}{ Population } & Nombre de pecheeurs & 41 & $4 t$ & 41 & 41 & 40 & 40 & 40 & 40 & 40 & 40 & 65 & 66 & 67 & 66 \\
\hline & & $\begin{array}{l}\text { Effort effectif total } \\
\text { (Nombre de jours) }\end{array}$ & 3034 & 2910 & 2910 & & 2000 & 1640 & 2000 & 2760 & 2800 & 3200 & 5005 & 4224 & 4422 & 4224 \\
\hline & & $\begin{array}{l}\text { Production totale } \\
\text { (Tonnes) }\end{array}$ & 49.2 & 80.8 & 58.2 & 53.7 & 28 & 32 & 24.4 & 26 & 29.1 & 25.3 & 41 & 31.4 & 39.1 & 16.6 \\
\hline & & P.U.E (KB / jour) & 16.3 & 27.7 & 20 & & 14 & 19.5 & 12.2 & 10.8 & 10.5 & 9 & 8.2 & 7.4 & 8.8 & 3.9 \\
\hline \multirow{5}{*}{$\begin{array}{l}\text { IXdiCATEURS } \\
\text { HALIEUTIOUES } \\
\text { DE } \\
\text { L'EXPLOITATION }\end{array}$} & Echantillon & Nombre de pêchears & 30 & 30 & 19 & 19 & 15 & 15 & 10 & 8 & 10 & 8 & 14 & 13 & 15 & 15 \\
\hline & \multirow{4}{*}{ Population } & Nombre de pecheurs & 654 & 655 & 655 & 529 & 461 & 388 & 386 & 389 & 337 & 324 & 242 & 233 & 221 & 220 \\
\hline & & $\begin{array}{l}\text { Effort effectif total } \\
\text { (Nombre de jours) }\end{array}$ & 14388 & 15720 & 15720 & & 12447 & 10088 & 8878 & 10503 & 9436 & 5832 & 5324 & 5370 & 9945 & 8860 \\
\hline & & $\begin{array}{l}\text { Production totafe } \\
\text { (Tonnes) }\end{array}$ & 237 & 324 & 274 & 69.6 & 52.3 & 50 & 40.1 & 19.2 & 53.3 & 7.7 & 39 & 16.7 & 24.9 & 25.1 \\
\hline & & P.U.E. $\left(\mathrm{Kg}_{\mathrm{g}} /\right.$ jour $)$ & 19.7 & 22.6 & 17 & & 4.8 & 4.3 & 3.3 & 1 & 4.3 & 1 & 7.4 & 3 & 2.5 & 2.9 \\
\hline
\end{tabular}


La 3 e période 1989 - 1992 a vu les captures totales du métier civelle-pibalour remonter en liaison avec un effort effectif total en hausse du fait de l'augmentation du nombre de pratiquants ; les P.U.E. se sont stabilisées au niveau de l'année 1988 et ont été au plus bas en 1992. Les P.U.E. du métier civelle-tamis sont restées à un niveau bas, très fluctuantes, de même que les captures totales, alors que l'effort effectif total a eu tendance à augmenter du fait de la multiplication du nombre de jours de pêche individuels (Tableau V).

Compte tenu de l'incertitude liée aux valeurs estimées des indicateurs halieutiques, dont les intervalles de confiance calculés pour la période 1989 - 1992 marquent l'amplitude, les analyses précédentes sont ramenées aux évolutions suivantes (fig. 4) :

- l'effort effectif total $f$ a diminué ou au plus est resté stable pour le métier civelle-tamis sur la période 1979 - 1992 ; l'effort effectif total f a diminué ou au plus est resté stable entre 1979 et 1988 et a augmenté par la suite pour le métier civelle-pibalour ;

- les captures totales $C$ ont diminué en 1982 - 1983 pour les deux métiers et ne sont remontées que pour le métier civelle-pibalour de 1989 à 1992 peut-être au plus au niveau de la période 1979 - 1981 ;

- les P.U.E. ont chuté en 1982 - 1983 pour les deux métiers et ont continué à diminuer par la suite ou au mieux sont restées au niveau de 1983.

Les évolutions de ces trois indicateurs halieutiques mises en parallèle pour chaque métier, permettent de diagnostiquer une réduction brutale de l'abondance des flux de civelles entre 1981 et 1983 . Cette abondance a eu tendance à décliner depuis ou au mieux s'est maintenue au même niveau bas jusqu'en 1992.

\subsubsection{Evolution inter-annuelle de l'abondance à court terme (1989 - 1992)}

L'analyse de l'évolution relative des indicateurs halieutiques est contrariée par les intervalles de confiance (fig. 4). Cependant, les tendances observées n'indiquent aucunement une reprise du recrutement estuarien en civelle.

\section{DISCUSSION}

\section{Le bilan de l'exploitation de la civelle en 1989}

Les résultats de production et de chiffre d'affaires obtenus pour l'année de référence 1989, confirment l'importance socio-économique de la pêche de la civelle en France.

L'augmentation du prix du produit (multiplié par 5 en francs constants entre 1979 et 1989) liée à la forte demande du marché espagnol a contribué à sauvegarder le chiffre d'affaires global de cette pêcherie, malgré le déclin des captures observé entre 1979 et 1989. Elle a permis le maintien et même parfois l'amélioration du chiffre d'affaires individuel des marins-pêcheurs en bateau depuis 1983, le maintien ou l'atténuation de la baisse du chiffre d'affaires des professionnels fluviaux et elle a sans doute contribué à la pérennisation d'un braconnage lucratif important.

En 1981, la civelle avec une valeur estimée de 77 millions de francs se trouvait à la $4^{e}$ place des ressources du Golfe de Gascogne après le merlu, la langoustine et la sole (ISTPM, 1983). En 1989, avec une valeur de plus de 200 millions de francs, elle passe à la $3^{\text {e }}$ place après le merlu et la sole, après rectification des états statistiques du CRTS (6) de la Rochelle.

La diversité des sources d'information, le décalage dans le temps des données utilisées, entraînent bon nombre d'incertitudes sur les effectifs de pêcheurs surtout nonprofessionnels et les productions dans certains secteurs de pêche. La méthode utilisée conduit cependant au premier bilan socio-économique sur l'exploitation de la civelle en France, mettant en jeu les données les plus fiables existantes sur l'ensemble des zones d'exploitation. Ce bilan correspond à des demandes réitérées du CIEM (7), de l'EIFAC (8) et

(6) CRTS : Centre Régional de Traitement Statistique des Affaires Maritimes de la Rochelle ;

(7) CIEM : Conseil International pour l'Exploration de la Mer ;

(8) EIFAC : European Inland Fisheries Advisory Commission. 

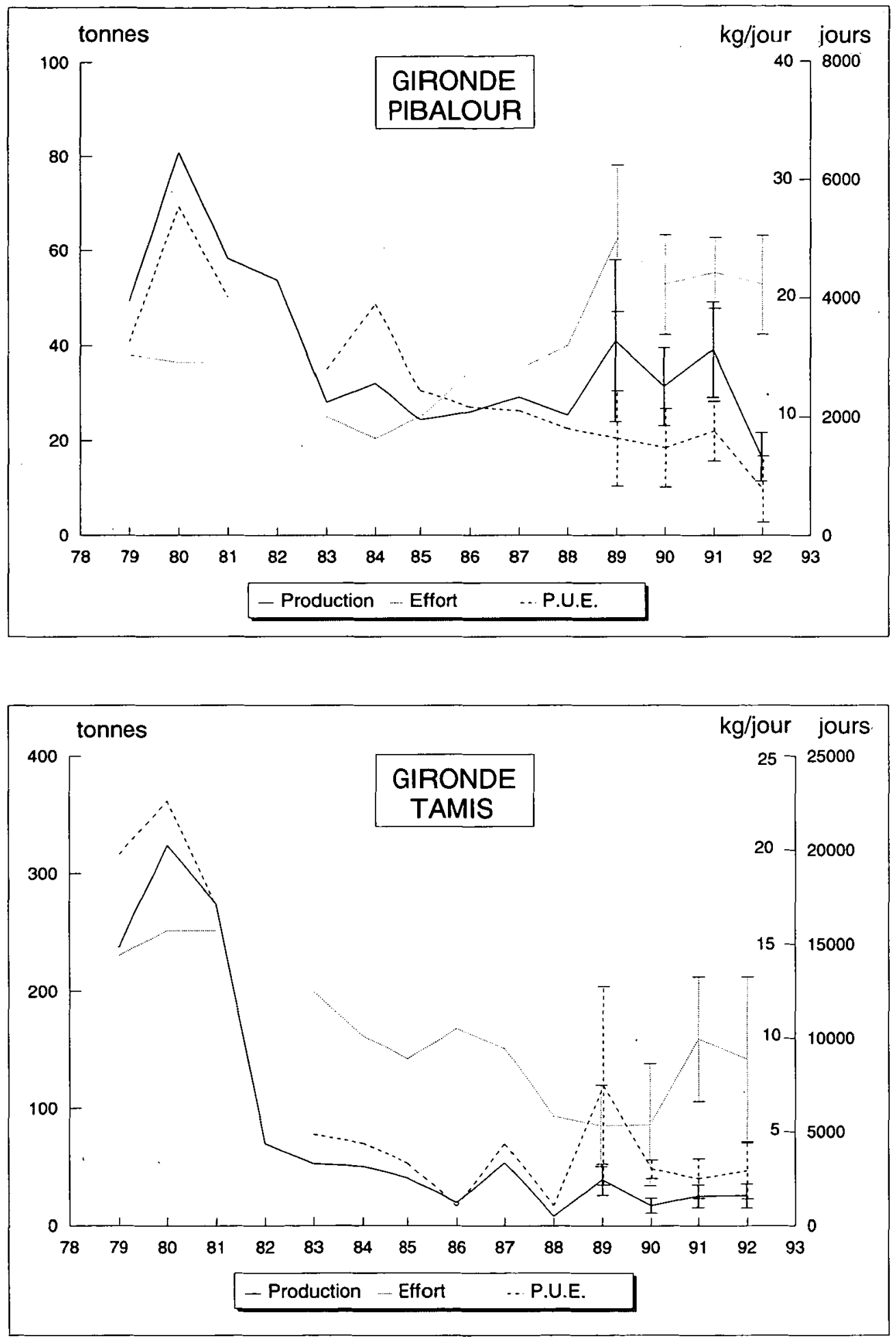

Figure 4 : Évolution des indicateurs halieutiques dans le bassin de la Gironde de 1979 à 1992 ( $l$ = intervalle de confiance des estimations, sécurité $95 \%$ ).

Figure 4 : Evolution of the fishing indicators in the Gironde basin from 1979 to 1992 ( 1 = confidence interval, security $95 \%$ ). 
du GRISAM (9). Il est unique en Europe ; aucun des pays exploitant la civelle à une échelle importante (Espagne, Portugal, Maroc, Grande-Bretagne) n'a produit d'état statistique récent concernant les effectifs de pêcheurs, les sites de pêche, les techniques de pêche utilisées et les productions.

\section{Diagnostic sur l'abondance}

La qualité des données halieutiques de base obtenues auprès des pêcheurs (bassin de la Gironde) ou du circuit commercial (bassins de la Vilaine et de la Loire) déterminent la pertinence des indicateurs halieutiques.

Sur le bassin de la Gironde par exemple, le taux d'échantillonnage est relativement faible : les pêcheurs coopératifs représentent selon les années de 8 à $18 \%$ de l'effectif des pêcheurs professionnels pour le métier civelle-pibalour et de 4 à $18 \%$ pour le métier civelle-tamis. Les données sont cependant assez fiables et représentatives de la variabilité intra et inter-annuelle des situations de pêche et des résultats de pêche.

La variabilité intra-annuelle des captures et des efforts effectifs individuels est à l'origine de l'amplitude élevée des intervalles de confiance des trois indicateurs $f, C$ et P.U.E. calculés pour la période 1989 - 1992. Cette variabilité est due à des différences de capturabilité dans toutes ses composantes (accessibilité, vulnérabilité et efficacité) qui s'expliquent pour chaque métier, par :

- métier civelle-pibalour :

- les performances (efficacité) des navires des marins-pêcheurs sur l'estuaire qui, selon leur type et la puissance motrice associée, poussent des pibalours de 5 à $14 \mathrm{~m}^{2}$ de surface totale;

- la disponibilité (accessibilité et vulnérabilité) de la ressource civelle influencée par les conditions hydrodynamiques et physico-chimiques locales (ELIE et ROCHARD, 1994, ce numéro) ; cette disponibilité semble augmenter d'aval en amont dans l'estuaire (CANTRELLE, 1981);

- métier civelle-tamis :

- les stratégies de pêche (efficacité) des pêcheurs professionnels au tamis sur les zones mixtes de Garonne - Dordogne - Isle, qui consistent d'une part, au cours d'une saison, soit à rester sur le même fleuve, soit à en changer périodiquement et d'autre part, au cours d'une marée, soit à pêcher à poste fixe, soit à se déplacer d'un poste de pêche à un autre ;

- la disponibilité de la ressource civelle qui semble perturbée par les mouvements des bateaux des nombreux pratiquants qui sillonnent des territoires de pêche limités dans les zones mixtes.

Pour le métier civelle-tamis, une grande inconnue réside dans l'effort réel et la production des non-professionnels. Les chiffres d'effort produits au tableau $V$ pour les nonprofessionnels correspondent aux pêcheurs licenciés civelle. Les quotas de licences ayant été diminués progressivement à partir de 1983, on peut supposer qu'un nombre important de ces pêcheurs qui n'ont pu obtenir une licence, a continué à pêcher et que la population de non-professionnels s'est maintenue à peu près au niveau de 1983. Les organisations de pêcheurs professionnels et les gardes-pêche avancent d'ailleurs actuellement le chiffre de 400 participants sur l'eau en pleine saison de pêche à la civelle.

Un effort identique à l'année 1983 (461 pratiquants licenciés) déployé pendant toute la période 1983 - 1992 entraînerait par rapport à l'effort théorique en diminution du tableau $\mathrm{V}$, un niveau moyen de production totale du métier civelle-tamis égal à $60-80 \mathrm{t}$, ce qui ne change rien aux termes du diagnostic sur l'abondance.

D'ailleurs, en reconsidérant les P.U.E. du métier civelle-tamis, on s'aperçoit que leur stabilité apparente à partir de 1983, est peut-être due aux données d'effort entrant dans

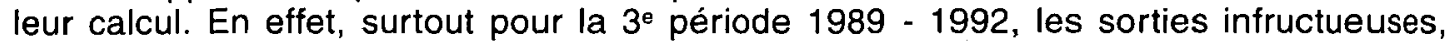


nombreuses, ne sont pas systématiquement notées par les pêcheurs coopératifs. L'effort en nombre de jours de pêche calculé est minoré et les valeurs des P.U.E. apparaissent de ce fait vraisemblablement un peu plus importantes qu'elles ne sont.

Ce problème d'évaluation de l'effort et de la production réelle des non-professionnels se retrouve dans d'autres secteurs où l'activité de pêche à la civelle est importante, par exemple : bassin de l'Adour où comme dans le bassin de la Gironde, les nonprofessionnels utilisent la même technique de pêche que les professionnels; bassin de la Loire où les techniques mises en oeuvre (tamis à main) par les non-professionnels limitent leur pouvoir de capture par rapport aux professionnels (drossage).

Les données halieutiques peuvent être biaisées par des variations inter-annuelles de capturabilité susceptibles de fournir une image plus ou moins déformée (biomasse apparente) de l'évolution inter-annuelle des flux de civelles. Par exemple, l'étude des six dernières saisons (1985 à 1990) dans les bassins de la Vilaine et de la Loire, a effectivement mis en évidence des modifications importantes de capturabilité dans toutes ses composantes, liées à l'influence :

- de l'hydroclimat (caractérisé par des situations environnementales extrêmes telles que les hivers rigoureux 1985, 1986 et 1987, les crues importantes de 1988 et les situations d'étiage de 1989 et 1990) sur la dynamique de la migration anadrome (accessibilité variable) et le comportement de la civelle (vulnérabilité) d'après DESAUNAY et al. (1987), GUERAULT et DESAUNAY (1989), GUERAULT et al. (1991) ;

- du comportement du pêcheur (efficacité) dont la fréquence des sorties, déterminée par les conditions de marché (gain escompté plutôt que quantité minimale pêchée) peut gonfler artificiellement l'effort de pêche (le résultat au niveau de l'indice d'abondance P.U.E. est ici l'opposé de celui des zones mixtes de Garonne - Dordogne-Isle : les P.U.E. apparaissent vraisemblablement un peu moins importantes qu'elles ne sont).

Ces variations inter-annuelles de capturabilité peuvent être importantes dans des systèmes ouverts comme ceux de la Loire, de la Gironde et de l'Adour. Elles sont par contre limitées dans l'estuaire de la Vilaine où la migration anadrome est bloquée par un barrage et où la stratégie d'exploitation, grâce à deux tamis à long manche (jusqu'à $8 \mathrm{~m}$ ), permet de s'adapter aux variations environnementales et à la distribution de la civelle dans la colonne d'eau (ELIE et RIGAUD, 1984).

Ainsi, les deux indicateurs $C$ et P.U.E. de cet estuaire s'accordent pour montrer que le recrutement annuel a pu être divisé par 3 ou 4 entre la fin des années 70 et celle des années 80 dans l'estuaire de la Vilaine.

Un schéma identique se dégage de l'examen des indicateurs halieutiques du bassin de la Loire (estuaire et zones mixtes, un seul métier) et du bassin de la Gironde (estuaire et zones mixtes, deux métiers), où le recrutement annuel a pu être divisé dans les mêmes proportions.

Cette convergence des résultats sur ces trois bassins autorise à penser que l'on a assisté, au début des années 80 , à une chute de l'abondance des flux de civelles dans les eaux intérieures qui ne s'est pas démentie depuis, même si les dernières années 1993 et 1994 sont marquées par une remontée des captures moyennes pour les pêcheurs en bateau pratiquant dans les estuaires.

II faut aussi signaler que la puissance de pêche et donc l'effort réel a augmenté depuis la fin des années 70 dans les estuaires. Pour les bateaux poussant des tamis, la puissance motrice moyenne est passée de 31 CV en 1974 (ELIE, 1979) à 62 CV en 1986 (calculs effectués à partir des résultats de CIPE, 1986). Pour les bateaux poussant des pibalours, la puissance motrice moyenne était de $70 \mathrm{CV}$ en 1986 , avec $46 \%$ des bateaux à moins de $60 \mathrm{CV}, 45 \%$ avec une puissance motrice comprise entre 60 et $100 \mathrm{CV}$ et $9 \%$ avec une puissance motrice supérieure à $100 \mathrm{CV}$ (origine des données identique à celle des bateaux-tamis). Cette puissance motrice s'est maintenue et a sans doute augmenté depuis 1986, en même temps que les surfaces des pibalours (certains petits pibalours ayant été remplacés par des plus grands). L'effort réel a donc manifestement augmenté dans les estuaires entre les années 70 et les années 80 , ce qui tend à consolider le diagnostic porté sur l'évolution de l'abondance des flux de civelles. 


\section{CONCLUSION}

Nous avons montré que la pêcherie de civelle en France représente encore, malgré une chute de la production au début des années 80 , une activité socio-économique de première importance, par le chiffre d'affaires qu'elle dégage : l'espèce anguille était placée au deuxième rang en valeur des productions du Golfe de Gascogne en 1989.

Le diagnostic proposé de diminution d'abondance de la civelle, phénomène commun aux trois bassins étudiés de la façade atlantique, ne doit pas être considéré comme une certitude mais seulement comme une forte présomption du fait des limites des investigations (nombre de sites et durée des suivis) et des limites d'interprétation (fiabilité des données halieutiques et corrélation avec les phénomènes biologiques).

Cependant, cette présomption se confirme dans tous les résultats enregistrés dans d'autres pays d'Europe (Suède, Norvège, Irlande, Angleterre, Danemark, Pays-Bas, Allemagne) et la période de diminution des stades civelle, anguille jaune et anguille argentée observée, coïncide avec celle mise en évidence en France pour la civelle.

Le statut d'espèce menacée de l'anguille demande sans aucun doute à être précisé, vérifié, mais l'exigence légitime de démonstration irréfutable d'un phénomène, dont les implications socio-économiques n'échappent à personne, ne saurait être un frein au principe de précaution qui implique, comme l'avait préconisé le Groupe National Anguille (ANONYME, 1984) :

- des investigations scientifiques sur la dynamique des stocks d'anguilles à l'échelle de bassins-versants ateliers coordonnés au niveau européen ;

— des actions d'amélioration de l'habitat et de circulation de l'espèce pour tous ses stades biologiques ;

— des aménagements de la filière pêche de la civelle en ce qui concerne l'effort de pêche et les circuits de commercialisation ;

— un suivi statistique des pêches opérationnel, garantissant la fiabilité des indicateurs halieutiques et permettant d'intégrer les pêcheurs dans un processus de gestion de la ressource.

L'établissement du présent bilan, qu'il serait souhaitable de renouveler à l'horizon 2000, a reposé sur des suivis statistiques dont seuls ceux concernant le bassin de la Gironde et le bassin de l'Adour se maintiennent actuellement.

Les suivis de référence devraient être réactivés, pérennisés et étendus à d'autres bassins ateliers représentatifs du territoire national, car ils sont indispensables aux administrations qui ont à gérer l'activité de pêche fluvio-estuarienne et aux organismes scientifiques qui ont besoin, souvent, de séries chronologiques longues pour poser un diagnostic sérieux sur l'évolution des ressources dites "renouvelables".

\section{BIBLIOGRAPHIE}

ANONYME, 1984. Rapport de synthèse et programme quinquennal. Réunions Banyuls, Nantes, La Rochelle, 1983 - 1984. Groupe National Anguille, 60 p.

AUBRUN L., 1986. Inventaire de l'exploitation de l'anguille sur le littoral de la Bretagne. ENSA Rennes. Publ. Dép. Halieut. $n^{\circ} 1,107$.

AUBRUN L., 1987. Inventaire de l'exploitation de l'anguille sur le littoral Sud-Gascogne. ENSA Rennes. Publ. Dép. Halieut. $n^{\circ} 5,143 p$.

BABIN D., 1993. Contribution à l'étude d'une ressource naturelle renouvelable : la pêche professionnelle en eau douce en France. Thèse $3^{\mathrm{e}}$ cycle CEMAGREF/Univ. Rennes II, $300 \mathrm{p}$.

CANTRELLE I., 1981. Etude de la pêche et de la migration des civelles (Anguilla anguilla L.) dans l'estuaire de la Gironde. Thèse d'océanographie biologique, Univ. Paris VI, $237 p$. 
CASTELNAUD G., 1978. Etude de la pêche aux filets et aux engins dans l'estuaire de la Gironde. Thèse $3^{\circ}$ cycle. CEMAGREF Bordeaux, Division ALA/Univ. Bordeaux III, $189 \mathrm{p}$.

CASTELNAUD G., TROUVERY M., de VERDILHAC Ph., 1981. La pêche des poissons migrateurs dans le bassin Garonne-Dordogne. 2. Evaluation de la production au moyen de carnets de pêche. Actes du XXVle Congrès A.F.L., Orléans, juin 1981, SRAE éd., p. 242-246.

CASTELNAUD G., CEREZUELLE D., GUCHAN A. ROCHARD E. 1985. La pêche des migrateurs en Gironde : $2^{e}$ partie : Enquête socio-professionnelle : propositions de gestion. CEMAGREF Bordeaux, Division ALA/MSH Aquitaine/Ministère de l'Environnement/Département de la Gironde, $110 \mathrm{p}$.

CASTELNAUD G., BABIN D., 1990. Analysis of the professional fishery in the Loire-Allier and Vilaine basins, France, Proceeding Symposium EIFAC on Management of Freshwater fisheries, Goteborg, Sweden, 31 may - 3 june 1988, p. 151-167.

CASTELNAUD G., BABIN D., 1992. La pêche professionnelle fluviale et lacustre en France. Enquête au fil de l'eau. Coll. Etudes. Ressources en eau, $n^{\circ} 5$, CEMAGREF/Division. ALA, Bordeaux, $291 \mathrm{p}$.

CASTELNAUD G., ROCHARD E., 1994. Surveillance halieutique de l'estuaire de la Gironde. Suivi statistique. 1992, étude de la faune circulante 1993. Rapp. CEMAGREF de Bordeaux/EDF, $161 \mathrm{p}$.

C.I.P.E., 1982. La filière civelle. Doc. Edition FIOM/Comité Central des Pêches Maritimes Paris, Juin 1982, $151 \mathrm{p}$.

C.I.P.E., 1983. Annexe $n^{\circ}$ I au P.V. du 27/09/1983. Edition Comité Central des Pêches Maritimes, Paris, $1 \mathrm{p}$.

C.I.P.E., 1986. Annexe $n^{\circ} V$ au P.V. du 26/06/1986. Annexe $n^{\circ} \mid$ au P.V. du 8/10/1986. Edition Comité Central des Pêches Maritimes, Paris, 1 p. et $8 \mathrm{p}$.

DAUBREE L., DROUIN DE BOUVILLE R. de, 1900. Pêche fluviale en France. Principaux engins et modes de pêche autorisés ou interdits. Ministère de l'agriculture, Administration des eaux et des forêts. Exposition universelle, Paris 1900, Imprimerie Nationale, Paris, $652 \mathrm{p}$.

DESAUNAY Y., 1987. Inventaire de l'exploitation de l'anguille sur le littoral Manche-Est. Rapp. int. IFREMER, DRV $87018 \mathrm{RH} / \mathrm{Nantes}, 36 \mathrm{p}$.

DESAUNAY Y., GUERAULT D., BEILLOIS P., 1987. Dynamique de la migration anadrome de la civelle (Anguilla anguilla) dans l'estuaire de la Loire : rôle des facteurs climatiques vis-à-vis de la pêche et du recrutement. Cons. int. Explor. Mer, C.M. 1987, $M: 18,22 \mathrm{p}$.

DESAUNAY Y., AUBRUN L., 1988. Description des pêcheries d'anguille (Anguilla anguilla) sur le littoral français de la Manche et de l'Atlantique. Cons. int. Explor. Mer, C.M. $1988, M: 25,15 \mathrm{p}$.

ELIE P., 1979. Contribution à l'étude des montées de civelles d'Anguilla anguilla L. (poisson téléostéen anguilliforme) dans l'estuaire de la Loire : pêche, écologie, écophysiologie et élevage. Thèse $3^{e}$ cycle, Univ. Rennes, $372 \mathrm{p}$.

ELIE P., FONTENELLE G., 1982. La pêche de la civelle d'Anguilla anguilla en France. Nécessité d'une gestion de cette ressource. Actes Colloque International des Entretiens Ecologiques de Dijon, p. 112-124.

ELIE P., RIGAUD C., 1984. Etude de la population d'anguilles de l'estuaire et du bassin versant de la Vilaine : pêche, biologie, écologie. Examen particulier de l'impact du barrage d'Arzal sur la migration anadrome (civelles). Tomes II et III, CEMAGREF de Bordeaux, Fac. de Rennes/URFDAAPP, $175 \mathrm{p}$.

ELIE P., ROCHARD E., 1994. Migration des civelles d'anguilles (Anguilla anguilla L.) dans les estuaires, modalités du phénomène et caractéristiques des individus. Bull. Fr. Pêche et Piscic., 335. 
GASCUEL D., 1987. La civelle d'anguille dans l'estuaire de la Sèvre Niortaise: Biologie, Ecologie, Exploitation. Publ. Dép. Halieut., ENSA Rennes, $n^{\circ}$ 4/1, 355 p, n 4/2, 204 p.

GUERAULT D., DESAUNAY Y., 1989. Evolution de l'abondance de la civelle (Anguilla anguilla) dans les estuaires de la Loire et de la Vilaine (France) 1977-1988. Working Group on eel/EIFAC, Porto, $20 \mathrm{p}$.

GUERAULT D., PROUZET P., DESAUNAY Y., BEILLOIS P., 1991. The recent evolution of the glass eel immigration in three French Atlantic Estuaries. Working Group on eel/EIFAC, Dublin, $18 \mathrm{p}$.

GUERAULT D., DESAUNAY Y., BEILLOIS P., 1993. La pêche de l'anguille dans l'estuaire de la Loire en 1989. Rapp. Int. IFREMER, DRV - 93/029 - RH/Nantes, 28 p.

GUERAULT D., DESAUNAY Y., BEILLOIS P., 1994. La pêche professionnelle des poissons migrateurs dans l'estuaire de la Loire en 1989. Repères Océan, IFREMER, $n^{\circ}$ 6, p. 3-28.

I.S.T.P.M., 1983. Réflexions sur les besoins en matière de recherche sur les pêches et les cultures marines. Rapports des groupes régionaux ou sectoriels. Partie golfe de Gascogne. Rapp. Technique $n^{\circ} 3 \mathrm{~b}, 16 \mathrm{p}$.

POPELIN E., 1971. Etude sur la pêche de l'anguille. Rapp. Ministère de l'Agriculture, Paris, Administration du GREF, $55 \mathrm{p}$.

PROUZET P., MARTINET J. P. et CUENDE F. X., 1994. Les pêches estuariennes du bassin de l'Adour de 1985 à 1991. Repères Océan, IFREMER nº, p. 33.70

ROCHARD E., 1992. Mise au point d'une méthode de suivi de l'abondance des amphihalins dans le système fluvio-estuarien de la Gironde, application à l'étude écobiologique de l'esturgeon Acipenser sturio. Thèse $3^{e}$ cycle CEMAGREF/Univ. Rennes, $306 \mathrm{p}$.

SERTIER M., ELIE P., ROCHARD E., 1990. Etude de suivi halieutique de l'estuaire de la Gironde 1989. Rapp. CEMAGREF de Bordeaux/EDF, $230 \mathrm{p}$. 